\title{
Periodic quotients of hyperbolic and large groups
}

\author{
Ashot Minasyan, AlexanderYu. Olshanskii and Dmitriy Sonkin
}

\begin{abstract}
Let $G$ be either a non-elementary (word) hyperbolic group or a large group (both in the sense of Gromov). In this article we describe several approaches for constructing continuous families of periodic quotients of $G$ with various properties.

The first three methods work for any non-elementary hyperbolic group, producing three different continua of periodic quotients of $G$. They are based on the results and techniques, that were developed by Ivanov and Olshanskii in order to show that there exists an integer $n$ such that $G / G^{n}$ is an infinite group of exponent $n$.

The fourth approach starts with a large group $G$ and produces a continuum of pairwise non-isomorphic periodic residually finite quotients.

Speaking of a particular application, we use each of these methods to give a positive answer to a question of Wiegold from the Kourovka Notebook.
\end{abstract}

Mathematics Subject Classification (2000). 20F50, 20 F67.

Keywords. Hyperbolic groups, large groups, periodic quotients.

\section{Introduction}

Let $G$ be an infinite finitely generated group. For $n \in \mathbb{N}$, by $G^{n}$ we denote the (normal) subgroup generated by the $n$-th powers of elements of $G$. Recall that $G$ is called periodic if each element of $G$ has a finite order. The group $G$ is said to be of bounded exponent if there is $n \in N$ such that $x^{n}=1$ for all $x \in G$, or, in other words, $G^{n}=\{1\}$; the least integer $n$ for which this equality holds is called the exponent of $G$. If for a periodic group $G \operatorname{such} n$ does not exist, it is said to be periodic of unbounded exponent. A group is called elementary if it has a cyclic subgroup of finite index.

The question whether the quotient group $G / G^{n}$ (which is of bounded exponent $n$ ) can be infinite has a long history in Group Theory. In the case when $G=F_{m}$ is a non-abelian free group, it was asked by W. Burnside in 1902 [8] and answered in the affirmative much later by P. Novikov and S. Adian [30] (see also [1]) for any sufficiently large odd exponent $n$. Yet later, A. Olshanskii [32] suggested a different, geometric approach to this problem, and, afterwards, in [33], applied similar 
methods to show that for every non-elementary torsion-free hyperbolic (in the sense of M. Gromov [18]) group $G$ there exists an integer $n \in \mathbb{N}$ such that $G / G^{n}$ is infinite. In [19] $\mathrm{S}$. Ivanov proved that $F_{m} / F_{m}^{n}$ is infinite for any sufficiently large even exponent $n$ divisible by $2^{9}$ (infiniteness of groups $F_{m} / F_{m}^{n}$ for large even values of $n$ was also proved by I. Lysënok [27]). Finally, in [20] Ivanov and Olshanskii combined the techniques from [33] and [19] and obtained the following theorem:

Theorem A. For every non-elementary hyperbolic group $G$ there exists a positive even integer $n=n(G)$ such that the following is true:

(a) The quotient group $G / G^{n}$ is infinite.

(b) Suppose that $n=n_{1} n_{2}$, where $n_{1}$ is odd and $n_{2}$ is a power of 2 . Then every finite subgroup of $G / G^{n}$ is isomorphic to an extension of a finite subgroup $K$ of $G$ by a subgroup of the direct product of two groups one of which is a dihedral group of order $2 n_{1}$ and the other is a direct product of several copies of a dihedral group of order $2 n_{2}$.

Remark 1. As it was shown in [20], Sec. 3, the exponent $n$ from the previous statement can be chosen to be any sufficiently large integer which is divisible by $2^{k_{0}+5} n_{0}$, where

a) $n_{0} / 2$ is the least common multiple of the exponents of the holomorphs $\operatorname{Hol}(K)$ over all finite subgroups $K$ of $G$;

b) $k_{0}$ is the minimal integer with $2^{k_{0}-3}>\max |K|$ over all finite subgroups $K$ of $G$.

The question which now naturally arises concerns the number (up to isomorphism) of periodic quotients for a fixed $G$.

In [31] the second author of the present article proved that there exists a continuum of 2-generated non-isomorphic groups with the property that every proper subgroup is cyclic of prime order. In [10] G. Deryabina showed that for every odd prime $p$ there is continuum of non-isomorphic simple groups, generated by two elements, such that all their proper subgroups are cyclic $p$-groups.

First examples of infinite finitely generated periodic residually finite groups were found by E. Golod in [13]. The techniques that he used allow, in fact, to obtain uncountably many of such groups (we thank L. Bartholdi for pointing this out). Explicitly, constructions of continuous families of finitely generated periodic residually finite groups first appear in the articles [15], [16] of R. Grigorchuk (see also [29], 2.10.5).

Speaking of periodic groups of bounded exponent, one should mention a result of V. Atabekyan [3], who demonstrated that the free group $F_{2}$, of rank 2, possesses a continuum of pairwise non-isomorphic simple quotient-groups of given period $n$ for each sufficiently large odd $n$. The third author of the present article established an analogous result in the situation when $n$ is a sufficiently large even integer divisible by $2^{9}$ (see [38]). 
The first objective of this article is to present several ways in which one can construct periodic quotients of a given non-elementary hyperbolic group $G$. We suggest three different methods each of which gives a continuum of such non-isomorphic quotients.

The second and the third approaches rely heavily on the techniques and statements used by Ivanov and Olshanskii in their proof of Theorem A in [20]. The first approach also uses their result but in a less thorough manner. These approaches are used to produce the following three theorems. (Recall that a group $H$ is said to be a central extension of the group $K$ if there exists a subgroup $N \triangleleft H$, contained in the center $Z(H)$ of $H$, such that $H / N \cong K$.)

Theorem 1. Let $G$ be a non-elementary hyperbolic group and let an integer $n$ be chosen according to Remark 1 . Then $G$ possesses a continuum of pairwise nonisomorphic periodic quotients $Q_{1 i}, i \in I_{1}$, of exponent $2 n$, each of which is a central extension of the group $G / G^{n}$.

Theorem 2. Let $G$ be a non-elementary hyperbolic group and let an integer $n$ be chosen according to Remark 1. Then $G$ possesses a continuum of pairwise nonisomorphic periodic quotients $Q_{2 i}, i \in I_{2}$, of unbounded exponent such that for every $i \in I_{2}$ the order of an arbitrary element $g \in Q_{2 i}$ divides $2^{l} n$ for some $l=l(g) \in \mathbb{N}$.

Every non-elementary hyperbolic group $G$ contains a unique maximal finite normal subgroup $E(G)$ ([34], Prop. 1). The quotient $\hat{G}=G / E(G)$, in addition to being non-elementary and hyperbolic, also satisfies $E(\hat{G})=\{1\}$.

Theorem 3. Assume that $G$ is a non-elementary hyperbolic group and denote $\hat{G}=G / E(G)$. Choose a positive integer $\hat{n}$ arising after an application of Remark 1 to $\hat{G}$. Then $G$ has a continuum of pairwise non-isomorphic centerless quotients of exponent $2 \hat{n}$.

Observe that if $Z(K)=\{1\}$ and $H_{1}, H_{2}$ are two central extensions of $K$ then $H_{1} / Z\left(H_{1}\right) \cong K \cong H_{2} / Z\left(H_{2}\right)$.

We will show that if the original group $G$ is centerless, then so is the group $G / G^{n}$. Thus the collections of quotients produced by Theorem 1 and Theorem 3 are significantly different because for any $i, j \in I_{2}$ one has $Q_{1 i} / Z\left(Q_{1 i}\right) \cong Q_{1 j} / Z\left(Q_{1 j}\right)$.

Our second objective is to obtain an analogue of the above results for large groups. Recall that a group $G$ is said to be large if it has a normal subgroup $N \triangleleft G$ of finite index, for which there exists an epimorphism $\varphi: N \rightarrow F$, where $F$ is a non-abelian free group. A recent result of M. Lackenby [25] asserts that if $G$ is a finitely generated large group and $g_{1}, \ldots, g_{r} \in G$, then the quotient $G /\left\langle\left\langle g_{1}^{n}, \ldots, g_{r}^{n}\right\rangle\right\rangle$ is large for infinitely many $n \in \mathbb{N}$. Lackenby's proof is topological. A different, combinatorial, proof of this result (not requiring the large group to be finitely generated) was found by A. Olshanskii and D. Osin in [37]; we will employ their methods to prove Theorem 4 below. 
Theorem 4. Suppose that $G$ is a finitely generated group having a normal subgroup $N$ of finite index that maps onto a non-abelian free group. Let $p$ be an arbitrary prime number. Then $G$ possesses $2^{\aleph_{0}}$ of periodic residually finite pairwise non-isomorphic quotients $H_{j}, j \in J$, such that for every $j \in J$ the natural image of $N$ in $H_{j}$ is a p-group.

The output of the previous theorem is very different from the continuous families given by Theorems 1,2, 3 as it produces periodic quotients with the additional property of residual finiteness. We recall that an infinite periodic finitely generated group of bounded exponent cannot be residually finite due to E. Zelmanov's positive solution of the restricted Burnside problem [39]-[40]. In the authors' opinion, the periodic quotients from the claim of Theorem 2 are not very likely to be residually finite. For instance, if $G$ is a non-elementary hyperbolic group possessing no proper finite index subgroups (it was shown I. Kapovich and D. Wise [22] and, independently, by the second author [36] that the existence of such a group is equivalent to the existence of a non-residually finite hyperbolic group, which is a well-known open problem), then no non-trivial quotient of $G$ can be residually finite. Thus an application of Theorem 2 to $G$ will produce a continuum of non-(residually finite) periodic groups.

Let $G$ be a finitely generated group and $p \in \mathbb{N}$ be a prime number. Define a series of finite index characteristic subgroups $\delta_{i}^{p}(G)$ of $G$ by $\delta_{0}^{p}(G)=G$ and

$$
\delta_{i}^{p}(G)=\left[\delta_{i-1}^{p}(G), \delta_{i-1}^{p}(G)\right]\left(\delta_{i-1}^{p}(G)\right)^{p} \leq \delta_{i-1}^{p}(G), \quad i \in \mathbb{N} .
$$

If $G$ is finitely generated, then for every prime $p$ and $t \in \mathbb{N}$, the number $\left|G / \delta_{t}^{p}(G)\right|$ is a natural invariant of $G$. Another point in which the fourth approach differs from the others is that it uses these invariants in order to distinguish between the quotients of $G$. Thus we show that for every prime $p$, there exist continually many finitely generated residually finite $p$-groups that can be distinguished via natural inner algebraic invariants.

Let $\Omega=\{0,1\}^{\mathbb{N}}$ be the set of all infinite sequences of 0 's and 1's. In Section 8 we prove

Corollary 1. Assume that $G$ is a finitely generated group, $N \triangleleft G$ is a normal subgroup mapping onto $F_{2}$ and $G / N$ is a finite $p$-group for some prime $p \in \mathbb{N}$. Then for every $\omega \in \Omega, G$ has quotient-group $H_{\omega}$ such that

(a) $H_{\omega}$ is a periodic p-group;

(b) $\bigcap_{i=0}^{\infty} \delta_{i}^{p}\left(H_{\omega}\right)=\{1\}$, thus $H_{\omega}$ is residually finite;

(c) if $\omega \neq \omega^{\prime} \in \Omega$ then there is $t \in \mathbb{N}$ such that $\left|H_{\omega} / \delta_{t}^{p}\left(H_{\omega}\right)\right| \neq\left|H_{\omega^{\prime}} / \delta_{t}^{p}\left(H_{\omega^{\prime}}\right)\right|$; consequently $H_{\omega} \nsucceq H_{\omega^{\prime}}$.

Finally, after combining Corollary 1 with a theorem of B. Baumslag and S. Pride [5], we deduce 
Corollary 2. Assume that a group $G$ admits a presentation with $k \geq 2$ generators and at most $k-2$ relators. Then for any prime $p, G$ has a collection of quotients $\left\{H_{\omega} \mid \omega \in \Omega\right\}$ such that

- $H_{\omega}$ is a periodic p-group for every $\omega \in \Omega$;

- $H_{\omega}$ is residually finite for every $\omega \in \Omega$;

- if $\omega \neq \omega^{\prime} \in \Omega$ then there is $t \in \mathbb{N}$ such that $\left|H_{\omega} / \delta_{t}^{p}\left(H_{\omega}\right)\right| \neq\left|H_{\omega^{\prime}} / \delta_{t}^{p}\left(H_{\omega^{\prime}}\right)\right|$; consequently $H_{\omega} \not H_{\omega^{\prime}}$.

\section{A particular application}

Before proving Theorems 1-4 we are going to consider one application. We will show that each of these theorems can be used to answer the following question of J. Wiegold:

Question ([24], 16.101). Does the group $G=\left\langle x, y \mid x^{2}=y^{4}=(x y)^{8}=1\right\rangle$ have uncountably many quotients each of which is a 2 -group?

As mentioned by Wiegold, the group $G$ can be homomorphically mapped onto a 2-group $\Gamma$ that is a subgroup of index 2 in the first Grigorchuk's group $\mathcal{E}$ (introduced in [14]). It is known that every proper quotient of $\mathcal{E}$ is finite [17], in other terms, $\mathcal{E}$ is just infinite. In a private communication with the first author, R. Grigorchuk asserted that a similar property holds for $\Gamma$. Thus, the group $\Gamma$ has only countably many distinct quotients. (More generally, L. Bartholdi has recently proved that every finitely generated subgroup of Grigorchuk's group $\mathcal{E}$ has at most countably many distinct quotients [4].)

In order to apply our approaches to the group $G$, we need to observe that $G$ is a subgroup of index 2 in the group

$$
T=\left\langle a, b, c \mid a^{2}=b^{2}=c^{2}=1,(a b)^{2}=(b c)^{4}=(a c)^{8}=1\right\rangle .
$$

And since $1 / 2+1 / 4+1 / 8<1, T$ is a reflection group of isometries of the hyperbolic plane $\mathbb{H}^{2}$, whose fundamental domain is a triangle with angles $\pi / 2, \pi / 4$ and $\pi / 8$. In fact, $G$ consists of all isometries from $T$ preserving the orientation on $\mathbb{H}^{2}$ (see [11], 7.3.1).

Hence the group $G$ acts properly discontinuously and cocompactly on $\mathbb{H}^{2}$, therefore it is non-elementary (word) hyperbolic. It is well known that every finite subgroup of $T$ lies inside of a finite parabolic subgroup [6], Ex. 2d, p. 130. Hence, it must be a subgroup of a dihedral group $D(2 k)$ of order $2 k$, where $k=2,4$ or 8 . Since $G$ contains no reflections, every finite subgroup of $G$ will be cyclic of order dividing 8 . Now, if $K$ is a cyclic group of order $2^{m}, m \in \mathbb{N}$, then $|\operatorname{Aut}(K)|=2^{m-1}$, hence $\operatorname{Hol}(K)=K \rtimes \operatorname{Aut}(K)$ has order $2^{2 m-1}$. By Remark 1, the exponent $n$ from the 
assumptions of Theorems 1 and 2 can be chosen as a sufficiently large power of 2 . Therefore both of these theorems give an affirmative answer to Wiegold's question.

By a classical theorem of E. Cartan [9], p. 267, (see also [7], Ch. II.2, Cor. 2.8) every finite subgroup $K$ of isometries of $\mathbb{H}^{2}$ fixes a non-empty subset that is convex in a strong sense: for any two distinct points fixed by $K, K$ will fix the entire bi-infinite geodesic in $\mathbb{H}^{2}$ passing through these points. The fixed-point set of a normal subgroup is invariant under the action of the entire group, hence the fixed-point set $\operatorname{Fix}(E(G))$ of $E(G)$ cannot consist of a single point (because $G$ is infinite and acts properly discontinuously). By the same reason, it cannot be a bi-infinite geodesic. Therefore, $E(G)$ fixes at least three points in general position, hence $\operatorname{Fix}(E(G))=\mathbb{H}^{2}$. And since the action of $G$ is faithful, one can conclude that $E(G)=\{1\}$. Thus Theorem 3 will produce a continuum of pairwise non-isomorphic centerless quotients of $G$ of exponent $2 n$ (for the same $n$ as above).

It remains to show that Theorem 4 can also be applied to $G$. It is well known that hyperbolic triangle groups, such as $G$, are large ([11], 7.3.1). For our purposes, however, we will need to exhibit a stronger property, namely, the existence of a normal subgroup $N \triangleleft G$, mapping homomorphically onto a non-abelian free group such that $|G: N|=2^{l}$ for some $l \in \mathbb{N}$.

Denote by $A=\langle x\rangle_{2} *\langle y\rangle_{4}$ the free product of cyclic groups of orders 2 and 4 . Let $B$ be the cartesian subgroup of $A$, i.e., the kernel of the natural homomorphism from $A$ onto the direct product $\langle x\rangle_{2} \times\langle y\rangle_{4}$. The cartesian subgroup of a free product is a free group (this follows from Kurosh subgroup theorem and the fact that the cartesian subgroup trivially intersects the free factors). Note that $B$ is freely generated by its elements $[x, y],\left[x, y^{2}\right]$ and $\left[x, y^{3}\right]$ (where $[u, v]=u v u^{-1} v^{-1}$ ). Set $C=B^{2}$ to be the normal subgroup of $A$ generated by the squares of elements of $B$. Then $|B: C|=8,(x y)^{4}=[x, y]\left[x, y^{2}\right]^{-1}\left[x, y^{3}\right] \in B \backslash C$ and $(x y)^{8} \in C$. By Schreier's formula, $C$ is a free group of rank $(3-1) 8+1=17$. Since $|A:\langle x y\rangle C|=8$, we can use [37], Lemma 2.3, to show that $D=\left\langle\left\langle(x y)^{8}\right\rangle\right\rangle^{A} \triangleleft C$ is a normal closure in $C$ of at most 8 elements. Hence the subgroup $E=C / D \triangleleft A / D=G$ has a presentation with 17 generators and 8 relators. Since $17-8 \geq 2$, by a result of Baumslag and Pride [5], for every sufficiently large $k \in \mathbb{N}, E$ has a normal subgroup $N^{\prime}$ of index $k$ such that $N^{\prime}$ can be mapped onto a non-abelian free group. Of course, one can take $k=2^{l^{\prime}}$ for some $l^{\prime} \in \mathbb{N}$. Observe that $N=\bigcap_{g \in G} g N^{\prime} g^{-1} \triangleleft G$ has finite index in $N^{\prime}$, and, therefore, it can also be mapped onto non-abelian free group. It remains to note that $E / N$ is a finite 2-group because $g N^{\prime} g^{-1} \triangleleft E$ and $\left|E: g N^{\prime} g^{-1}\right|=2^{l^{\prime}}$ for every $g \in G$. Consequently $|G: N|=|G: E| \cdot|E: N|=2^{6} \cdot|E: N|=2^{l}$ for some $l \in \mathbb{N}$.

Therefore an application of Theorem 4 to $G$ and $N$ provides a continuum of pairwise non-isomorphic residually finite quotients of $G$ each of which is a 2-group.

One can now see that Wiegold's problem mentioned above can be solved in a number different ways. In the course of writing this article, R. Grigorchuk informed the authors of yet another possible method to solve this problem, using the techniques developed in his article [15]. 


\section{Preliminaries}

Fix a group $G$ with a finite symmetrized generating set $\mathcal{A}$. If $g \in G,|g|_{\mathcal{A}}$ will denote the length of a shortest word $W$ over $\mathcal{A}$ representing $g$ in $G$. This gives rise to the standard left-invariant distance function $d(\cdot, \cdot)$ on $G$ defined by $d(x, y)=\left|x^{-1} y\right|_{\mathcal{A}}$ for any $x, y \in G$. Afterwards this can be extended to the metric $d(\cdot, \cdot)$ on the Cayley graph $\Gamma(G, \mathcal{A})$ in the usual way. For subset a $Q$ of $\Gamma(G, \mathcal{A})$, the closed $\varepsilon$-neighborhood is defined by

$$
\mathcal{N}_{\varepsilon}(Q)=\{x \in \Gamma(G, \mathcal{A}) \mid \text { there exists } y \in Q \text { such that } d(x, y) \leq \varepsilon\} .
$$

For any two points $x, y \in \Gamma(G, \mathcal{A}),[x, y]$ will denote a geodesic segment between them.

Fix a number $\delta \geq 0$. A geodesic $n$-gon in $\Gamma(G, \mathcal{A})$ is called $\delta$-slim if each of its sides is contained in the closed $\delta$-neighborhood of the others. Using the definition of E. Rips, we will say that $G$ is $\delta$-hyperbolic if every geodesic triangle in its Cayley graph is $\delta$-slim. As a consequence of this definition, it is easy to obtain that each geodesic quadrilateral in $\Gamma(G, \mathcal{A})$ is $2 \delta$-slim. Further on we shall assume that the group $G$ is non-elementary and $\delta$-hyperbolic for some given $\delta \geq 0$. There is a number of other (equivalent up to changing $\delta$ ) definitions of $\delta$-hyperbolicity - see [2], for example. Since some of the Lemmas quoted below utilize them, we will suppose that our $\delta$ is sufficiently large so that $G$ also satisfies all of these other definitions.

The length of a word $W$ over the alphabet $\mathcal{A}$ will be denoted by $\|W\|$. Suppose that $W$ represents an element $g \in G$. Then we define $|W|_{\mathcal{A}}=|g|_{\mathcal{A}}$. If $p$ is a path in the Cayley graph, then $p_{-}, p_{+}$and $\|p\|$ will denote its starting point, ending point and the length, respectively. In the case when $p$ is a simplicial path, $\operatorname{lab}(p)$ will stand for the word written on $p$ and $p^{-1}-$ for the inverse path to $p$, that is, $p_{-}^{-1}=p_{+}$, $p_{+}^{-1}=p_{-}$and $\operatorname{lab}\left(p^{-1}\right) \equiv \operatorname{lab}(p)^{-1}$ Given some numbers $\bar{\lambda}, \bar{c}$ satisfying $0<\lambda \leq 1$, $c \geq 0$, we will say that $p$ is $(\lambda, c)$-quasigeodesic if for any subpath $q$ of $p$ one has $\lambda\|q\|-c \leq d\left(q_{-}, q_{+}\right)$. A word $W$ will be called $(\lambda, c)$-quasigeodesic provided some (equivalently, any) path $p$, with $\operatorname{lab}(p) \equiv W$, is $(\lambda, c)$-quasigeodesic in $\Gamma(G, \mathcal{A})$.

The next two lemmas are standard properties of a hyperbolic metric space:

Lemma 1 ([12], 5.6, 5.11, [2], 3.3). There is a constant $v=v(\delta, \lambda, c)$ such that for any $(\lambda, c)$-quasigeodesic path $p$ in $\Gamma(G, \mathcal{A})$ and a geodesic $q$ with $p_{-}=q_{-}$, $p_{+}=q_{+}$, one has $p \subset \mathcal{N}_{v}(q)$ and $q \subset \mathcal{N}_{v}(p)$.

Lemma 2 ([28], Lemma 4.1). Consider a geodesic quadrilateral $x_{1} x_{2} x_{3} x_{4}$ in the Cayley graph $\Gamma(G, \mathcal{A})$ with $d\left(x_{2}, x_{3}\right)>d\left(x_{1}, x_{2}\right)+d\left(x_{3}, x_{4}\right)$. Then there are points $u, v \in\left[x_{2}, x_{3}\right]$ such that $d\left(x_{2}, u\right) \leq d\left(x_{1}, x_{2}\right), d\left(v, x_{3}\right) \leq d\left(x_{3}, x_{4}\right)$ and the geodesic subsegment $[u, v]$ of $\left[x_{2}, x_{3}\right]$ lies $2 \delta$-close to the side $\left[x_{1}, x_{4}\right]$.

It is well known (see, for example, [34]) that in a hyperbolic group $G$ every element $g$ of infinite order is contained in a unique maximal elementary subgroup 
$E_{G}(g)$, and

$$
\begin{aligned}
E_{G}(g) & =\left\{x \in G \mid x g^{m} x^{-1}=g^{n} \text { for some } m, n \in \mathbb{Z} \backslash\{0\}\right\} \\
& =\left\{x \in G \mid x g^{n} x^{-1}=g^{ \pm n} \text { for some } n \in \mathbb{N}\right\} .
\end{aligned}
$$

The subgroup $E_{G}^{+}(g)=\left\{x \in G \mid x g^{n} x^{-1}=g^{n}\right.$ for some $\left.n \in \mathbb{N}\right\}$ has index at most 2 in $E_{G}(g)$.

\section{Central extensions}

Fix a presentation $\left\langle\mathcal{A} \mid R \in \mathcal{R}_{0}\right\rangle$ of the initial non-elementary hyperbolic group $G=G(0)$, where the set of generators $\mathcal{A}$ is finite and $\mathcal{R}_{0}$ is the set of all relators in $G$, that is, $\mathcal{R}_{0}$ consists of all words from over the alphabet $\mathcal{A}^{ \pm 1}$ that represent the identity element in $G$.

Choose the exponent $n$ by Theorem A and consider the group $G(\infty)=G / G^{n}$.

Let us recall a few things from [20]. It is shown that

$$
G(\infty)=\left\langle\mathcal{A} \mid R, R \in \mathcal{R}_{0}, A_{1}^{n}, A_{2}^{n}, \ldots\right\rangle,
$$

where the word $A_{j}, j \in \mathbb{N}$, is called the period of rank $j$. Moreover, each group

$$
G(i):=\left\langle\mathcal{A} \mid R, R \in \mathcal{R}_{0}, A_{1}^{n}, A_{2}^{n}, \ldots, A_{i}^{n}\right\rangle
$$

of rank $i$ is also non-elementary hyperbolic. For every $i \in \mathbb{N}$, the word $A_{i}$ has infinite order in $G(i-1)$ and there exists a unique maximal finite subgroup $\mathscr{F}\left(A_{i}\right)$ which is normalized by $A_{i}$ in $G(i-1)$ (more precisely, $\mathcal{F}\left(A_{i}\right)$ is the torsion subgroup of $\left.E_{G(i-1)}^{+}\left(A_{i}\right)\right)$. One of the properties, proved in [20], states that $A_{i}^{n / 2} T A_{i}^{-n / 2} T^{-1}=1$ in $G(i-1)$ for any $i \in \mathbb{N}$ and any word $T$ representing an element of $\mathcal{F}\left(A_{i}\right)$ (see the adaptation of Lemma 18.5 from [19] in [20], Section 14).

A word $J$ is called an $\mathscr{F}\left(A_{i}\right)$-involution if $J$ normalizes the subgroup $\mathscr{F}\left(A_{i}\right)$ of $G(i-1), J^{2} \in \mathcal{F}\left(A_{i}\right)$ and $J^{-1} A_{i} J=A_{i}^{-1} T$ for some $T \in \mathscr{F}\left(A_{i}\right)$. By [20] and [19], Lemma 19.2, $A_{i}^{n / 2} J A_{i}^{n / 2} J^{-1}=1$ in the group $G(i-1)$. If for a given $i \in \mathbb{N}$ there exists an $\mathcal{F}\left(A_{i}\right)$-involution, the period $A_{i}$ is said to be even; otherwise, $A_{i}$ is said to be odd. Equivalently, $A_{i}$ is odd if and only if $E_{G(i-1)}\left(A_{i}\right)=E_{G(i-1)}^{+}\left(A_{i}\right)$.

Consider a diagram $\Delta$ over the presentation (2) on some orientable surface $S$ (say, a plane or a sphere). Then one can fix the same (say, clockwise) direction of the contours of each cell in $\Delta$. If $\Pi$ is a cell corresponding to the relation $A_{j}^{n}=1$, $j \in \mathbb{N}$, one defines its rank $r(\Pi)$ to be $j$; by definition, $r(\Pi)=0$ if $\Pi$ corresponds to a relator $R \in \mathcal{R}_{0}$. A vertex $o \in \partial \Pi$ is called a phase vertex if, starting at $o$ and going along the contour of $\Pi$ in the clockwise direction, we read the word $A_{j}^{ \pm n}$. The number $r(\Delta)=\max \{r(\Pi) \mid \Pi \in \Delta\}$ is called the strict rank of the diagram $\Delta$. If the strict rank of $\Delta$ is $i$, the type $t(\Delta)$ of $\Delta$ is a sequence $\left(\tau_{i}, \tau_{i-1}, \ldots, \tau_{0}\right)$ where $\tau_{j}$ 
is the number of cells of rank $j$ in $\Delta$. For the convenience of inductive reasoning we will impose the short-lex order on the set of all types.

Let $\Pi_{k}, k=1,2$, be two distinct cells of $\Delta$ of rank $j$. The pair of cells $\Pi_{1}$ and $\Pi_{2}$ is said to be a reducible $j$-pair if there is a simple path $t$ in the diagram $\Delta$, connecting two phase vertices $o_{1}$ and $o_{2}$ on the their boundary contours, such that one of the following holds:

1) The period $A_{j}$ is even and the words written on $\partial \Pi_{1}$ and $\partial \Pi_{2}$ starting with the vertices $o_{1}$ and $o_{2}$ are the same (i.e., $A_{j}^{ \pm n}$ ), and $\operatorname{lab}(t)$ is an $\mathscr{F}\left(A_{j}\right)$-involution.

2) The words written on $\partial \Pi_{1}$ and $\partial \Pi_{2}$ starting with $o_{1}$ and $o_{2}$ are mutually inverse (that is, they are either $A_{j}^{n}$ and $A_{j}^{-n}$ or $A_{j}^{-n}$ and $A_{j}^{n}$, respectively), and $\operatorname{lab}(t)$ represents an element of $\mathscr{F}\left(A_{j}\right)$ in $G(j-1)$.

In the case when the original group $G$ is free, the following statement can be compared with [35], Lemma 5.2.

Lemma 3. Let $\left(\tau_{i}, \tau_{i-1}, \ldots, \tau_{0}\right)$ be the type of an arbitrary spherical diagram $\Delta$ over the presentation (2). Then for and any $k \in\{1,2, \ldots, i\}$ the integer $\tau_{k}$ is even.

Proof. Fix $k \in\{1, \ldots, i\}$ and use induction on $t(\Delta)$. If $\Delta$ has no cells of positive rank the claim is trivial. Otherwise, the adaptation of [19], Lemma 6.2, described in Section 7 of [20] claims that $\Delta$ has a reducible $j$-pair of cells $\Pi_{1}$ and $\Pi_{2}$ for some $1 \leq j \leq i$. Let $t$ denote the corresponding simple path between some phase vertices $o_{1}$ and $o_{2}$ of the contours $\partial \Pi_{1}$ and $\partial \Pi_{2}$, and set $T \equiv \mathrm{lab}(t)$. Denote by $\Gamma$ the subdiagram of $\Delta$ consisting of $\Pi_{1}, \Pi_{2}$ and $t$. Then the word written on the boundary of $\Gamma$ starting $o_{1}$ is $A_{j}^{\epsilon n} T A_{j}^{\hat{\epsilon} n} T^{-1}$ where $\epsilon, \hat{\epsilon} \in\{1,-1\}$. By the definition of a reducible $j$-pair and the properties, mentioned above, in $G(j-1)$ we have

$$
A_{j}^{\epsilon n / 2} T A_{j}^{\hat{\epsilon} n / 2} T^{-1}=1 .
$$

By van Kampen's Lemma there exists a disk diagram $\Gamma^{\prime}$ over the presentation (2) with $r\left(\Gamma^{\prime}\right) \leq j-1$ whose boundary label is $A_{j}^{\epsilon n / 2} T A_{j}^{\hat{\epsilon} n / 2} T^{-1}$. Gluing $\Gamma^{\prime}$ with a copy of itself along the two different occurrences of $T$ on their boundaries one obtains a new disk diagram $\Gamma^{\prime \prime}$ (with $r\left(\Gamma^{\prime \prime}\right)=r\left(\Gamma^{\prime}\right)$ ), whose boundary label is letter-by-letter equal to $A_{j}^{\epsilon n} T A_{j}^{\hat{\epsilon} n} T^{-1}$ which is the boundary label of $\Gamma$. Obviously, the number $\chi_{k}$ of cells of rank $k$ in $\Gamma^{\prime \prime}$ is twice that number for $\Gamma^{\prime}$, and so is even.

Now one can perform a standard diagram surgery, by cutting $\Gamma$ out of $\Delta$ and replacing it with $\Gamma^{\prime \prime}$, to obtain a new spherical diagram $\Delta^{\prime}$. Since $t\left(\Delta^{\prime}\right)<t(\Delta)$ we can use the induction hypothesis to show that the number $\psi_{k}$ of cells of rank $k$ in $\Delta^{\prime}$ is even. Due to the construction, one has $\tau_{k}=\psi_{k}-\chi_{k}+2$ (if $k=j$ ) or $\tau_{k}=\psi_{k}-\chi_{k}$ (if $k \neq j$ ). In either case $\tau_{k}$ will be even.

Let $F$ be the free group on $\mathcal{A}$ and let $N_{0}$ and $N$ denote the the kernels of the natural homomorphisms $F \rightarrow G$ and $F \rightarrow G(\infty)$, respectively. The mutual commutator $[F, N]$ is the normal subgroup of $F$ generated by all commutators of the form $[x, y]$ where $x \in F$ and $y \in N$. 
Lemma 4. Suppose that the word $W \equiv A_{i_{1}}^{n \tau_{1}} A_{i_{2}}^{n \tau_{2}} \ldots A_{i_{s}}^{n \tau_{s}}, 1 \leq i_{1}<i_{2}<\ldots i_{s}$, represents an element of the subgroup $[F, N] N^{2} N_{0}$ in $F$. Then the integers $\tau_{1}, \ldots, \tau_{s}$ are all even.

Proof. Clearly, by the definition of the subgroups $[F, N]$ and $N^{2}$, one can write $W$ as a product $\prod_{t} U_{t} A_{j_{t}}^{ \pm n} U_{t}^{-1} h$ where $h \in N_{0}$, and for every $l$ the number of occurrences of $A_{l}^{ \pm n}$ in this product is even. Therefore, as in the proof of van Kampen's Lemma (see, for example, [26]), one can construct a disk diagram $\Gamma$ over the presentation (2), whose boundary contour is labelled by $W$ and which has an even number $\psi_{l}$ of cells of every positive rank $l$. On the other hand, the equality from the assumptions of the lemma gives rise to a diagram $\Delta_{2}$ over the presentation (2) with the same boundary contour and having exactly $\tau_{k}$ cells of rank $i_{k}$ for each $k=1,2, \ldots, s$. Gluing together $\Delta_{1}$ with a mirror copy of $\Delta_{2}$ along their boundary contours, one will obtain a spherical diagram $\Delta_{3}$. Applying Lemma 3 to $\Delta_{3}$ one gets that the number $\left(\tau_{k}+\psi_{i_{k}}\right)$ of the cells of rank $i_{k}$ in it is even. Therefore $\tau_{k}$ must also be even.

Set $L=G^{n} \triangleleft G$ and $M=[G, L] L^{2} \triangleleft G$; the full preimages of these subgroups in $F$ are $N$ and $[F, N] N^{2} N_{0}$, respectively. Then the group $G / M$ is a central extension of the group $G(\infty)=G / L$ by the central subgroup $L / M$. The analogue of the next theorem in the case when $G$ is a free group of finite rank was proved in [35], Thm. 5.

Corollary 3. The group $L / M$ is a direct product of the groups of order 2 generated by the natural images of $A_{j}^{n}, j \in \mathbb{N}$.

Proof. Evidently, $L / M$ is an abelian group generated by the images $\hat{a}_{j}$ of $A_{j}^{n}, j \in \mathbb{N}$, and these images have order at most 2 in it. Now, Lemma 4 asserts that if an element of the form $\hat{a}_{i_{1}}^{\tau_{1}} \ldots \hat{a}_{i_{s}}^{\tau_{s}}$ is trivial in $L / M$, then $\tau_{k}$ is even for each $k=1, \ldots, s$. Hence $L / M$ is a direct product of the subgroups $\left\langle\hat{a}_{j}\right\rangle$.

The following observation is a consequence of the fact that there can be at most countably many different homomorphisms from a given finitely generated group $G$ to a fixed countable group.

Remark 2. Let $G$ be a finitely generated group, $I$ be a set of cardinality continuum and $\left\{N_{i}\right\}_{i \in I}$ be a family of normal subgroups of $G$ such that $N_{i} \neq N_{j}$ whenever $i \neq j$. Then the set of quotients $\left\{G / N_{i}\right\}_{i \in I}$ contains continually many pairwise non-isomorphic groups.

Proof of Theorem 1. Clearly, the order of any element $g \in G / M$ divides $2 n$. It is known that an infinite hyperbolic group always contains an element of infinite order ([12], 8.3.36), hence $G(\infty) \neq G(i)$ for every $i \in \mathbb{N}$. Consequently the set of periods $\left\{A_{i}\right\}$ is infinite. Thus, according to Theorem 3 , the abelian group $L / M$ has continually many distinct subgroups. As $L / M$ is contained in the center of the 
group $H=G / M, H$ also has a continuum of different central normal subgroups $Z_{i}, i \in I$. Note that since $G$ is finitely generated, then so is $H$. By Remark 2 , there is a continuum of non-isomorphic groups among the quotients $\left\{H / Z_{i} \mid i \in I\right\}$, all of which are central extensions of $G(\infty)$. Therefore, the statement of the theorem is true.

\section{Quotients of unbounded exponent}

In the present section we will prove Theorem 2. Suppose that the non-elementary hyperbolic group $G$ has a presentation $\left\langle\mathcal{A} \mid R \in \mathcal{R}_{0}\right\rangle$, where $\mathcal{A}$ is finite and $\mathcal{R}_{0}$ is the set of all relators in $G$.

By [20], Lemma 18, there is a constant $k_{0} \in \mathbb{N}$ such that $2^{k_{0}-3}>\max |K|$ for any finite subgroup $K$ of $G$. By $n_{0}$ denote a constant such that $n_{0} / 2$ is the least common multiple of the exponents of holomorphs $\operatorname{Hol}(K)$ over all finite subgroups $K$ of $G$.

Let us recall the construction of the quotient $G / G^{n}$ from [20], where $n$ is a large integer divisible by $2^{k_{0}+5} n_{0}$.

Introduce a total order $\prec$ on the set of words in the alphabet $\mathcal{A}$ so that $\|X\|<\|Y\|$ implies $X \prec Y$. Set $G(0)=G$ and define groups $G(i)$ by induction on $i$.

As a matter of notational convenience, in what follows we shall often identify a word $W$ over the alphabet $\mathcal{A}$ with an element that it represents in $G(0)$. For a word $W$, representing an element of infinite order $w \in G(0)$, denote by $F(W)$ the maximal finite subgroup of $E^{+}(W)$. Such a $W$ is called simple in $G(0)$ (see [20], Sec. 4) if its coset in $E_{G}(W) / F(W)$ generates the cyclic subgroup $E_{G}^{+}(W) / F(W)$ and none of the conjugates of elements $W^{ \pm 1} F$ ( where the word $F$ represents an element from $F(W))$ has length less than $\|W\|$.

Assuming that the group $G(i-1), i \geq 1$, is already defined, consider the least (with respect to the order $\prec$ ) word $A$ of infinite order in $G(i-1)$, with the additional requirement that $A$ is simple in $G(0)$ if $\|A\|<C$ (see [20], Lemma 13, for the meaning of parameter $C$ ). Declare such a word $A$ to be the period $A_{i}$ of rank $i$, and define the group $G(i)$ by imposing the relation $A_{i}^{n}=1$ on the group $G(i-1)$ :

$$
G(i)=\left\langle\mathcal{A} \mid R \in \mathcal{R}_{0} \cup\left\{A_{1}^{n}, A_{2}^{n}, \ldots, A_{i}^{n}\right\}\right\rangle .
$$

The period $A_{i}$ exists for every $i \geq 1$, and the group $G / G^{n}$ is the direct limit of groups $G(i)$ :

$$
G / G^{n}=G(\infty)=\left\langle\mathcal{A} \mid R \in \mathcal{R}_{0} \cup\left\{A_{1}^{n}, A_{2}^{n}, \ldots\right\}\right\rangle .
$$

The construction of Ivanov and Olshanskii [20] may be modified in the following way. Instead of imposing on each step a relation $A^{n}=1$ for a fixed exponent $n$, we will introduce periodic relations with large exponent that may vary from step to step. In the case when $G(0)$ a non-abelian free group such modification has already appeared in [35]. 
Given a sequence $\omega=\left(\omega_{j}\right)_{j=1}^{\infty}$ of 0's and 1's, we modify the choice of defining relations for the groups $G(i)$ from [20] as follows.

Set $n(0)=n$ and, for $j \geq 1$, define $n(j)=\left(1+\omega_{j}\right) n(j-1)$. Inductively define groups $G_{\omega}(i)$ : set $G_{\omega}(0)=G$ and assuming that the period $A_{i}$ of rank $i$ is chosen, define $G_{\omega}(i)$ to be the quotient of $G_{\omega}(i-1)$ by the normal closure of $A_{i}^{n(i)}$ :

$$
G_{\omega}(i)=\left\langle\mathcal{A} \mid R \in \mathcal{R}_{0} \cup\left\{A_{1}^{n(1)}, A_{2}^{n(2)}, \ldots, A_{i}^{n(i)}\right\}\right\rangle .
$$

The direct limit of groups $G_{\omega}(i)$ with respect to canonical epimorphisms is denoted by $G_{\omega}(\infty)$.

Note that for any sequence $\omega$ of 0 's and 1 's the sequence $(n(j))$ is non-decreasing and $n(j) \geq n$ for every $j \geq 0$. We now refer to [35], and modify the arguments from [20] in a way similar to the one given in [35]. Namely, the order of the period $A_{i}$ in $G_{\omega}(\infty)$ is now $n(i)$ instead of $n$; in all the estimates, the terms $\left\|A_{i}^{n}\right\|$ and $n\left\|A_{i}\right\|$ are substituted by $\left\|A_{i}^{n(i)}\right\|$ and $n(i)\left\|A_{i}\right\|$, respectively; finite subgroups of $G_{\omega}(i)$ are isomorphically embedded into a direct product of a direct power of the dihedral group $D(2 n(i))$ (instead of $D(2 n))$ and a group elementary associated with $G$ (see [20]); $n$ is replaced by $n(i)$ in the group identities of the analogue of Lemma 15.10 [20],[19]; in the inductive step from $G_{\omega}(i)$ to $G_{\omega}(i+1)$ (in arguments from [19], $\S 18,19$, and their analogues from [20]) $n$ is replaced by $n(i+1)$.

After all of these modifications, we obtain that for any sequence $\omega$ the period $A_{i}$ exists for every $i \geq 1$ and the group $G_{\omega}(\infty)$ is infinite and periodic.

Let $\omega, \omega^{\prime}$ be two different sequences of 0 's and 1's. Then the kernels of the canonical homomorphisms $G \rightarrow G_{\omega}(\infty)$ and $G \rightarrow G_{\omega^{\prime}}(\infty)$ are different. Indeed, let $j$ be the smallest index where $\omega$ and $\omega^{\prime}$ differ, say $\omega_{j}=0, \omega_{j}^{\prime}=1$. We remark here that for all $i, 0 \leq i<j$, the sets of defining words of the groups $G_{\omega}(i)$ and $G_{\omega^{\prime}}(i)$ coincide in view of minimality of $j$ and the fact that the order $\prec$ was fixed a-priori. This means, in turn, that the same word $A_{j}$ is the period of rank $j$ in both $G_{\omega}(\infty)$ and $G_{\omega^{\prime}}(\infty)$. By the analogue of Lemma 10.4 [20] for $G_{\omega}(\infty)$ (for $G_{\omega^{\prime}}(\infty)$ ), the order of the period $A_{j}$ in $G_{\omega}(\infty)$ (in $G_{\omega^{\prime}}(\infty)$ ) is equal to $n(j)(2 n(j)$ respectively).

Thus, the groups $G_{\omega}(\infty)$ and $G_{\omega^{\prime}}(\infty)$ are quotients of the finitely generated group $G$ by different normal subgroups provided $\omega \neq \omega^{\prime}$. Remark 2 permits us to conclude that the set of pairwise non-isomorphic groups among $\left\{G_{\omega}(\infty)\right\}_{\omega}$ is of cardinality continuum.

\section{Aperiodic elements in hyperbolic groups}

The purpose of this section is to establish a few auxiliary results, that will be used in Section 7 in order to develop the third approach and prove Theorem 3.

Throughout this section we will assume that $G$ is a non-elementary hyperbolic group with a fixed finite symmetrized generating set $\mathcal{A}$. 
Let $W_{1}, W_{2}, \ldots, W_{l}$ be words in $\mathcal{A}$ representing elements $w_{1}, w_{2}, \ldots, w_{l}$ of infinite order, where $E_{G}\left(w_{i}\right) \neq E_{G}\left(w_{j}\right)$ for $i \neq j$. For any given $M \geq 0$ consider the set $S\left(W_{1}, \ldots, W_{l} ; M\right)$ consisting of words

$$
W \equiv W_{i_{1}}^{m_{1}} W_{i_{2}}^{m_{2}} \ldots W_{i_{s}}^{m_{s}},
$$

where $s \in \mathbb{N}, i_{k} \neq i_{k+1}$ for $k=1,2, \ldots, s-1$ (each $i_{k}$ belongs to $\{1, \ldots, l\}$ ), and $\left|m_{k}\right|>M$ for $k=2,3, \ldots, s-1$. The following lemma will be useful:

Lemma 5 ([34], Lemma 2.3). There exist constants $\lambda_{1}=\lambda_{1}\left(W_{1}, W_{2}, \ldots, W_{l}\right)>0$, $c_{1}=c_{1}\left(W_{1}, W_{2}, \ldots, W_{l}\right) \geq 0$ and $M_{1}=M_{1}\left(W_{1}, W_{2}, \ldots, W_{l}\right)>0$ such that any path $p$ in the Cayley graph $\Gamma(G, \mathcal{A})$ with $\operatorname{lab}(p) \in S\left(W_{1}, \ldots, W_{l} ; M_{1}\right)$ is $\left(\lambda_{1}, c_{1}\right)$ quasigeodesic.

Consider a closed path $p_{1} q_{1} p_{2} q_{2}$ in the Cayley graph $\Gamma(G, \mathcal{A})$ such that $\operatorname{lab}\left(q_{1}\right)$, $\operatorname{lab}\left(q_{2}^{-1}\right) \in S\left(W_{1}, \ldots, W_{l} ; M_{2}\right)$. Thus $q_{1}=o_{1} \ldots o_{s}$ where $\operatorname{lab}\left(o_{k}\right) \equiv W_{i_{k}}^{m_{k}}$, $i_{k} \in\{1, \ldots, l\}, k=1, \ldots, s$, and $i_{k} \neq i_{k+1}, k=1, \ldots, s-1$. Similarly, $q_{2}^{-1}=$ $\bar{o}_{1} \ldots \bar{o}_{\bar{s}}$ where $\operatorname{lab}\left(\bar{o}_{k}\right) \equiv W_{j_{k}}^{\bar{m}_{k}}, j_{k} \in\{1, \ldots, l\}, k=1, \ldots, \bar{s}$, and $j_{k} \neq j_{k+1}$, $k=1, \ldots, \bar{s}-1$. We will say that $v$ is a phase vertex of a path $o_{j}$ if the subpath of $o_{j}$ from $\left(o_{j}\right)_{-}$to $v$ is labelled by some power of the word $W_{i_{j}}$ (and similarly for $\bar{o}_{j}$ ).

Paths $o_{k}$ and $\bar{o}_{\bar{k}}$ will be called compatible if there is a path $u_{k}$ in $\Gamma(G, \mathcal{A})$ joining some phase vertices of $o_{k}$ and $\bar{o}_{\bar{k}}$ such that $\operatorname{lab}\left(u_{k}\right) W_{j_{\bar{k}}} \operatorname{lab}\left(u_{k}\right)^{-1}$ represents an element of $E_{G}\left(w_{i_{k}}\right)$ in $G$. Such a path $u_{k}$ is said to be matching.

The following is a simplification of [34], Lemma 2.5 (we note that instead of requiring $s$ and $\bar{s}$ to be bounded in the proof of [34] it is enough to demand that one uses only finitely many distinct words $W_{1}, \ldots, W_{l}$ ):

Lemma 6. Suppose that $q_{1}, q_{2}, p_{1}, p_{2}$ are as above, and $\left\|p_{1}\right\|,\left\|p_{2}\right\| \leq C_{1}$ for some $C_{1}$. Then there exist integers $M_{2}$ and $\epsilon \in\{-1,0,1\}$ such that the paths $o_{k}$ and $\bar{o}_{k+\epsilon}$ are compatible for all $k=2, \ldots, s-1$, whenever $\left|m_{1}\right|, \ldots,\left|m_{s}\right| \geq M_{2}$, $\left|\bar{m}_{1}\right|, \ldots,\left|\bar{m}_{\bar{s}}\right| \geq M_{2}$.

Recall (see[34]) that two elements $g, h$ having infinite order in $G$ are said to be commensurable if there exist $a \in G$ and $k, l \in \mathbb{Z} \backslash\{0\}$ such that $g^{k}=a h^{l} a^{-1}$.

Suppose now that the maximal finite normal subgroup $E(G)$ of a non-elementary hyperbolic group $G$ is trivial. An element $g \in G$ is said to be suitable if it has infinite order and $E_{G}(g)=\langle g\rangle$.

Lemma 7 ([34], Lemma 3.8). Every non-elementary hyperbolic group $G$ such that $E(G)=\{1\}$ contains infinitely many pairwise non-commensurable suitable elements.

A subgroup $H \leq G$ is called malnormal if $g \mathrm{Hg}^{-1} \cap H=\{1\}$ for all $g \in G \backslash H$. In the special case when $G$ is torsion-free, the following lemma was proved by I. Kapovich in [21], Thm. C. 
Lemma 8. Let $G$ be a non-elementary hyperbolic group with $E(G)=\{1\}$. Then there are words $W_{1}$ and $W_{2}$, representing elements of infinite order $w_{1}$ and $w_{2}$ in $G$, and constants $0<\lambda_{1} \leq 1, c_{1} \geq 0$ such that

- any path in $\Gamma(G, \mathcal{A})$ labelled by a word from $S\left(W_{1}, W_{2} ; 0\right)$ is $\left(\lambda_{1}, c_{1}\right)$-quasigeodesic;

- the subgroup $H=\left\langle w_{1}, w_{2}\right\rangle \leq G$ is free of rank 2 ;

- $H$ is malnormal in $G$.

Proof. By Lemma 7 one can find four pairwise non-commensurable suitable elements $w, x, y, z \in G$ represented by some words $W, X, Y, Z$ over the alphabet $\mathcal{A}$, respectively. Apply Lemma 5 to the set of words $S\left(W, X, Y, Z ; M_{1}\right)$ to find the constants $\lambda_{1}, c_{1}$ and $M_{1}$. Find $v_{1}=v_{1}\left(\delta, \lambda_{1}, c_{1}\right)$ according to Lemma 1 and denote $C_{1}=2 \delta+2 v_{1}$. Now let $M_{2}$ be the constant from the claim of Lemma 6 applied to any closed path $p_{1} q_{1} p_{2} q_{2}$ where $\operatorname{lab}\left(q_{i}\right) \in S\left(W, X, Y, Z ; M_{2}\right)$ and $\left\|p_{i}\right\| \leq C_{1}$, $i=1,2$. Fix an arbitrary integer $m>\max \left\{M_{1}, M_{2}\right\}$ satisfying $\lambda_{1} m-c_{1}>0$, and define $w_{1}=w^{m} x^{m}, w_{2}=y^{m} z^{m}$. Note that since the elements $w_{1}$ and $w_{2}$ are represented by the words $W_{1} \equiv W^{m} X^{m}$ and $W_{2} \equiv Y^{m} Z^{m}$ and $m>M_{1}$, every word from $S\left(W_{1}, W_{2} ; 0\right)$ belongs to $S\left(W, X, Y, Z ; M_{1}\right)$. Therefore the first claim of the lemma holds.

To show that $w_{1}$ and $w_{2}$ freely generate the subgroup $H=\left\langle w_{1}, w_{2}\right\rangle \leq G$ it is enough to check that any non-empty word $A \in S\left(W_{1}, W_{2} ; 0\right)$ is non-trivial in $G$. But this follows immediately from the $\left(\lambda_{1}, c_{1}\right)$-quasigeodesity:

$$
|A|_{G} \geq \lambda_{1}\|A\|-c_{1} \geq \lambda_{1} \min \left\{\left\|W_{1}\right\|,\left\|W_{2}\right\|\right\}-c_{1} \geq \lambda_{1} m-c_{1}>0 .
$$

Arguing by contradiction, assume that $H$ is not malnormal, i.e., $b a_{1} b^{-1}=a_{2}$ for some $b \in G \backslash H, a_{1}, a_{2} \in H \backslash\{1\}$; then $b a_{1}^{l} b^{-1}=a_{2}^{l}$ for all $l \in \mathbb{N}$. Let $A_{1}, A_{2} \in$ $S\left(W_{1}, W_{2} ; 0\right) \subset S\left(W, X, Y, Z ; M_{2}\right)$ and $B$ be some words representing $a_{1}, a_{2}$ and $b$. Observe that one can assume that the words $A_{1}$ and $A_{2}$ are cyclically reduced (with respect to $W_{1}$ and $W_{2}$ ) after replacing $B$ with some word $B_{1}$ representing an element $b_{1}$ of the double coset $H b H$; one will still have $b_{1} \notin H$ because $H b H \cap H=\emptyset$. Then the words $A_{1}^{l}$ and $A_{2}^{l}$ will also belong to $S\left(W, X, Y, Z ; M_{2}\right)$. Consider a closed path $p_{1}^{\prime} q_{1}^{\prime} p_{2}^{\prime} q_{2}^{\prime}$ in $\Gamma(G, \mathcal{A})$ such that $\operatorname{lab}\left(p_{1}^{\prime}\right) \equiv B_{1}, \operatorname{lab}\left(q_{1}^{\prime}\right) \equiv A_{1}^{l}, \operatorname{lab}\left(p_{2}^{\prime}\right) \equiv B_{1}^{-1}$, $\operatorname{lab}\left(q_{2}^{\prime}\right) \equiv A_{2}^{-l}$.

As the path $q_{1}^{\prime}$ is $\left(\lambda_{1}, c_{1}\right)$-quasigeodesic, one can take $l$ to be so large that it will have a subpath $q_{1}$ whose endpoints will be at distances greater than $\left(\left\|B_{1}\right\|+v_{1}\right)$ from the endpoints of $q_{1}^{\prime}$ and which will be labelled by one of the following words: $X^{m} Y^{m}$ (coming from an occurrence of the subword $W_{1} W_{2}$ in the middle of $A_{1}^{l}$ ), $X^{m} Z^{-m}$ (coming from an occurrence of the subword $W_{1} W_{2}^{-1}$ in the middle of $A_{1}^{l}$ ), $W^{-m} Y^{m}$ (from $W_{1}^{-1} W_{2}$ ), $W^{-m} Z^{-m}$ (from $W_{1}^{-1} W_{2}^{-1}$ ), $X^{m} W^{m}$ (from $W_{1} W_{1}$ ), $W^{-m} X^{-m}$ (from $W_{1}^{-1} W_{1}^{-1}$ ), $Y^{m} Z^{m}$ (from $W_{2} W_{2}$ ) and $Z^{-m} Y^{-m}$ (from $W_{2}^{-1} W_{2}^{-1}$ ). We shall consider only the first case, when $\operatorname{lab}\left(q_{1}\right) \equiv X^{m} Y^{m}$, because the other cases are completely similar. Thus, $q_{1}=o_{1} o_{2}$ where $\operatorname{lab}\left(o_{1}\right) \equiv X^{m}, \operatorname{lab}\left(o_{2}\right) \equiv Y^{m}$. 
By Lemma 1 there are points $\alpha_{1}, \alpha_{2} \in\left[\left(q_{1}^{\prime}\right)_{-},\left(q_{1}^{\prime}\right)_{+}\right]$such that $d\left(\alpha_{1},\left(q_{1}\right)_{-}\right) \leq v_{1}$ and $d\left(\alpha_{2},\left(q_{1}\right)_{+}\right) \leq v_{1}$. Then by Lemma 2 , applied to the geodesic quadrilateral with vertices $\left(p_{1}^{\prime}\right)_{-},\left(q_{1}^{\prime}\right)_{-},\left(p_{2}^{\prime}\right)_{-}$and $\left(q_{2}^{\prime}\right)_{-}$, one can find points $\beta_{1}, \beta_{2} \in\left[\left(p_{1}^{\prime}\right)_{-},\left(q_{2}^{\prime}\right)_{-}\right]$ satisfying $d\left(\alpha_{i}, \beta_{i}\right) \leq 2 \delta, i=1,2$. Applying Lemma 1 once again one will obtain points $\gamma_{1}, \gamma_{2} \in q_{2}^{\prime}$ with $d\left(\gamma_{i}, \beta_{i}\right) \leq v_{1}$. Let $q_{2}$ denote the subpath of $q_{2}^{\prime}\left(\right.$ or $\left.q_{2}^{\prime-1}\right)$ starting with $\gamma_{2}$ and ending with $\gamma_{1}$, and let $p_{1}=\left[\left(q_{2}\right)_{+},\left(q_{1}\right)_{-}\right], p_{2}=\left[\left(q_{1}\right)_{+},\left(q_{2}\right)_{-}\right]$. According to the triangle inequality one has

$$
\left\|p_{1}\right\|=d\left(\left(q_{2}\right)_{+},\left(q_{1}\right)_{-}\right) \leq d\left(\left(q_{1}\right)_{-}, \alpha_{1}\right)+d\left(\alpha_{1}, \beta_{1}\right)+d\left(\beta_{1}, \gamma_{1}\right) \leq 2 \delta+2 v_{1}=C_{1},
$$

and, similarly, $\left\|p_{2}\right\| \leq C_{1}$. Note that the labels of $q_{1}$ and $q_{2}^{-1}$ belong to the set $S\left(W, X, Y, Z ; M_{2}\right)$, hence by Lemma $6, q_{2}^{-1}$ contains subpaths $\bar{o}_{1}$ and $\bar{o}_{2}$ with $\left(\bar{o}_{1}\right)_{+}=\left(\bar{o}_{2}\right)_{-}, \operatorname{lab}\left(\bar{o}_{i}\right) \in\left\{W^{m}, X^{m}, Y^{m}, Z^{m}\right\}^{ \pm 1}, i=1,2$, such that there exist matching paths $u_{i}$ which connect some phase vertices of $o_{i}$ and $\bar{o}_{i}, i=1,2$. Since the elements $w, x, y, z$ are pairwise non-commensurable, by the definition of compatible paths one immediately gets $\operatorname{lab}\left(\bar{o}_{1}\right) \equiv X^{\epsilon_{1} m}$ and $\operatorname{lab}\left(o_{2}\right) \equiv Y^{\epsilon_{2} m}$ for some $\epsilon_{i} \in\{-1,1\}, i=1,2$. Moreover $\epsilon_{i}=1, i=1,2$, because $E_{G}(x)=E_{G}^{+}(x)=\langle x\rangle$ and $E_{G}(y)=E_{G}^{+}(y)=\langle y\rangle$. Thus the subpath $\bar{o}_{1} \bar{o}_{2}$ of $q_{2}^{-1}$ (and, hence, of $q_{2}^{\prime \pm 1}$ ) originates from an occurrence of the same subword $W_{1} W_{2}$ in $\operatorname{lab}\left(q_{2}^{\prime \pm 1}\right)$.

Now observe that since the word $\operatorname{lab}\left(u_{1}\right) X \operatorname{lab}\left(u_{1}\right)^{-1}$ represents an element of $\langle x\rangle$ then, applying (1), one achieves $\operatorname{lab}\left(u_{1}\right)=X^{t_{1}}$ in $G$ for some $t_{1} \in Z$. Similarly, $\operatorname{lab}\left(u_{2}\right)=Y^{t_{2}}$ in $G$ for some $t_{2} \in \mathbb{Z}$. Since the endpoints of $u_{1}$ and $u_{2}$ are phase vertices of $o_{1}, \bar{o}_{1}$ and $o_{2}, \bar{o}_{2}$, there are paths $r_{i}, i=1,2$, having the same starting point $\left(r_{i}\right)_{-}=\left(o_{1}\right)_{+}=\left(o_{2}\right)_{-}$and the same ending point $\left(r_{i}\right)_{+}=\left(\bar{o}_{1}\right)_{+}=\left(\bar{o}_{2}\right)_{-}$, $i=1,2$, such that $\operatorname{lab}\left(r_{1}\right)$ represents an element of $\langle x\rangle$ and $\operatorname{lab}\left(r_{2}\right)$ represents an element of $\langle y\rangle$. But $\langle x\rangle \cap\langle y\rangle=\{1\}$ and $\operatorname{lab}\left(r_{1}\right)=\operatorname{lab}\left(r_{2}\right)$ in $G$, therefore lab $\left(r_{1}\right)$ represents the identity element in $G$.

Let $q_{1}^{\prime \prime}, q_{2}^{\prime \prime}$ denote the subpaths of $q_{1}^{-1}$ and $q_{2}^{\prime-1}$, respectively, satisfying $\left(q_{1}^{\prime \prime}\right)_{-}=$ $\left(r_{1}\right)_{-},\left(q_{1}^{\prime \prime}\right)_{+}=\left(p_{1}^{\prime}\right)_{+},\left(q_{2}^{\prime \prime}\right)_{-}=\left(p_{1}^{\prime}\right)_{-}$and $\left(q_{2}^{\prime \prime}\right)_{+}=\left(r_{1}\right)_{+}$. By construction of the paths $o_{1}, o_{2}, \bar{o}_{1}, \bar{o}_{2}$, the words $\operatorname{lab}\left(q_{i}^{\prime \prime}\right), i=1,2$, belong to $S\left(W_{1}, W_{2} ; 0\right)$, and so the elements which they represent in $G$ belong to $H$. Finally, the equality $b_{1}=\operatorname{lab}\left(p_{1}^{\prime}\right)=\operatorname{lab}\left(q_{2}^{\prime \prime}\right) \operatorname{lab}\left(r_{1}^{-1}\right) \operatorname{lab}\left(q_{1}^{\prime \prime}\right)$ combined with $\operatorname{lab}\left(r_{1}^{-1}\right)=1$ imply that $b_{1} \in H$, contradicting the initial assumption. Thus the lemma is proved.

A word $B$ over $\mathcal{A}$ (and the element $b \in G$ represented by it) is said to be cyclically reduced if for any word $U$, conjugate to $B$ in $G$, one has $\|B\| \leq\|U\|$. For $m \in \mathbb{Z}$, any subword of $B^{m}$ is called $B$-periodic.

The following fact was established in [33], Lemma 27, for the case of a torsionfree hyperbolic group $G$ by Olshanskii; in [20], Lemma 12, Ivanov and Olshanskii noted that the claim continues to hold even if $G$ possesses elements of finite order.

Lemma 9. Assume that $G$ is a non-elementary hyperbolic group with a finite generating set $\mathcal{A}$. There exist numbers $0<\lambda_{0} \leq 1$ and $c_{0} \geq 0$ such that for every 
cyclically reduced word $B$ (over $A)$ ), representing an element of infinite order in $G$, any $B$-periodic word $V$ is $\left(\lambda_{0}, c_{0}\right)$-quasigeodesic.

Lemma 10. Let $G$ be a $\delta$-hyperbolic group and $\bar{\lambda}, \bar{c}$ be some numbers satisfying $0<\bar{\lambda} \leq 1$ and $\bar{c} \geq 0$. Then there exists a constant $\Lambda_{1}=\Lambda_{1}(\delta, \bar{\lambda}, \bar{c}) \geq 0$ such that the following holds.

Assume that $X_{1}, X_{2}$ are some words over $\mathcal{A}$ having infinite order in $G$ and for each $i=1,2$, any path in $\Gamma(G, \mathcal{A})$ labelled by a power of $X_{i}$ is $(\bar{\lambda}, \bar{c})$-quasigeodesic. If $X_{1}^{l}=A X_{2}^{k} A^{-1}$ in $G$ for some word $A$ and non-zero integers $k, l$, then one can find words $U, W$ and a $X_{1}$-periodic word $V$ such that $X_{2}=U V W$ in $G$ and $\|U\|,\|W\| \leq \Lambda_{1}$.

Proof. Let $\bar{v}=\bar{v}(\delta, \bar{\lambda}, \bar{c})$ be obtained from the claim of Lemma 1. Set $\Lambda_{1}=2 \delta+2 \bar{v}$ and denote by $a \in G$ the element represented by the word $A$.

By the assumptions, $X_{1}^{l s}=A X_{2}^{k s} A^{-1}$ in $G$ for every $s \in \mathbb{Z}$ (pick $s$ so that $k s>0)$. Consider the quadrilateral with sides $p_{1}, q_{1}, p_{2}$ and $q_{2}$ in $\Gamma(G, \mathcal{A})$ where $\left(p_{1}\right)_{-}=\left(q_{2}\right)_{+}=1, \operatorname{lab}\left(p_{1}\right) \equiv A,\left(q_{1}\right)_{-}=\left(p_{1}\right)_{+}, \operatorname{lab}\left(q_{1}\right) \equiv X_{2}^{k s},\left(p_{2}\right)_{-}=$ $\left(q_{1}\right)_{+}, \operatorname{lab}\left(p_{2}\right) \equiv A^{-1},\left(q_{2}\right)_{-}=\left(p_{2}\right)_{+}, \operatorname{lab}\left(q_{2}\right) \equiv X_{1}^{-l s}$. Since the paths $q_{1}$ and $q_{2}$ are $(\bar{\lambda}, \bar{c})$-quasigeodesic, if one takes $|s|$ to be sufficiently large (compared to $|a|_{\mathcal{A}}$ ), it will be possible to find a subpath $r$ of $q_{1}$ labelled by $X_{2}$ whose endpoints are at distances at least $\left(|a|_{\mathcal{A}}+\bar{v}\right)$ from the endpoints of $q_{1}$. According to Lemma 1 there are points $u, v$ lying on the geodesic segment $\left[\left(q_{1}\right)_{-},\left(q_{1}\right)_{+}\right]$at distances at most $\bar{v}$ from $r_{-}$and $r_{+}$, respectively. Because of the choice of the subpath $r$ one can now apply Lemma 2 to find points $u^{\prime}, v^{\prime} \in\left[\left(q_{2}\right)_{+},\left(q_{2}\right)_{-}\right]$such that $d\left(u, u^{\prime}\right) \leq 2 \delta$ and $d\left(v, v^{\prime}\right) \leq 2 \delta$. Again by Lemma 1 , there are points $u^{\prime \prime}, v^{\prime \prime} \in q_{2}$ situated at distances at most $\bar{v}$ from $u^{\prime}$ and $v^{\prime}$, respectively. Hence $d\left(r_{-}, u^{\prime \prime}\right), d\left(r_{+}, v^{\prime \prime}\right) \leq 2 \bar{v}+2 \delta=\Lambda_{1}$.

Now the claim of the lemma will hold if one denotes by $U$ and $W$ the words written on the geodesic paths $\left[r_{-}, u^{\prime \prime}\right]$ and $\left[v^{\prime \prime}, r_{+}\right]$, respectively, and by $V$ the word written on the subpath of $q_{2}$ (or $\left.q_{2}^{-1}\right)$ starting with $u^{\prime \prime}$ and ending with $v^{\prime \prime}$.

Suppose that $\Lambda>0$ and $t \in \mathbb{N}$. Following [20] we shall say that an element $g \in G$ is $(\Lambda, t)$-periodic if there are words $U, V$ and $W$ over $\mathcal{A}$ such that $g=U V W$ in $G, \max \left\{|U|_{\mathcal{A}},|V|_{\mathcal{A}}\right\} \leq \Lambda$, and $V$ is a $Z$-periodic word, for some cyclically reduced word $Z$ having infinite order in $G$, with $\|V\| \geq t\|Z\|$. An element $h \in G$ is called $(\Lambda, t)$-aperiodic provided that for every factorization $h=h_{1} h_{2} h_{3}$, where $h_{1}, h_{2}, h_{3} \in G$, such that $|h|_{\mathcal{A}}=\left|h_{1}\right|_{\mathcal{A}}+\left|h_{2}\right|_{\mathcal{A}}+\left|h_{3}\right|_{\mathcal{A}}$, the element $h_{2}$ is not $(\Lambda, t)$ periodic. It is not difficult to see that, in geometric terms, $g$ is $(\Lambda, t)$-aperiodic if and only if for any geodesic path $p$ in $\Gamma(G, \mathcal{A})$, whose label represents $g$ in $G$, and for any path $q$ such that $q_{-}, q_{+} \in \mathcal{N}_{\Lambda}(p)$ and $\operatorname{lab}(q) \equiv V$ is a $Z$-periodic word (for some cyclically reduced word $Z$ having infinite order in $G$ ), one has $\|q\|=\|V\|<t\|Z\|$.

Suppose that $W_{1}, W_{2}$ are some words over the alphabet $\mathcal{A}$ representing elements $w_{1}, w_{2} \in G$. Let $F^{+}\left(W_{1}, W_{2}\right)$ denote the free monoid generated by the words $W_{1}$ and $W_{2}$ (i.e., the set of all positive words in $W_{1}$ and $W_{2}$ ). Assume that for some $\lambda_{1}>0$ 
and $c_{1} \geq 0$, any word from $F^{+}\left(W_{1}, W_{2}\right)$ is $\left(\lambda_{1}, c_{1}\right)$-quasigeodesic in $G$ and that the canonical map $\psi: F^{+}\left(W_{1}, W_{2}\right) \rightarrow G$ is injective. Let $F^{+} \subset G$ denote the set of elements represented by the words from $F^{+}\left(W_{1}, W_{2}\right)$, i.e., $F^{+}=\psi\left(F^{+}\left(W_{1}, W_{2}\right)\right)$. Note that each non-trivial $f \in F^{+}$has infinite order in $G$.

Lemma 11. There is a constant $\Lambda_{1} \geq 0$ such that for any $\Lambda^{\prime} \geq \Lambda_{1}$ there exist an integer $t^{\prime}>0$ and infinitely many pairwise non-commensurable elements in $F^{+}$that are $\left(\Lambda^{\prime}, t^{\prime}\right)$-aperiodic in $G$.

Proof. Let $\lambda_{0}$ and $c_{0}$ be the constants from Lemma 9. Define $\bar{\lambda}=\min \left\{\lambda_{0}, \lambda_{1}\right\}$ and $\bar{c}=\max \left\{c_{0}, c_{1}\right\}$, find the corresponding constant $\Lambda_{1}=\Lambda_{1}(\delta, \bar{\lambda}, \bar{c}) \geq 0$ according to Lemma 10 , and take an arbitrary $\Lambda^{\prime} \geq \Lambda_{1}$.

In [33], Lemmas 30, 31, it was shown that there exists $t^{\prime} \in \mathbb{N}$ and an infinite subset $\mathcal{B}=\left\{b_{1}, b_{2}, \ldots\right\} \subset F^{+}$consisting of $\left(\Lambda^{\prime}, t^{\prime}\right)$-aperiodic elements in $G$ (the proof works in the general case of an arbitrary non-elementary hyperbolic group, as observed in [20], Lemma 15).

For each $j \in \mathbb{N}$ find the (unique) word $B_{j} \in F^{+}\left(W_{1}, W_{2}\right)$ with $\psi\left(B_{j}\right)=b_{j}$. As $W_{1}$ and $W_{2}$ are words over $\mathcal{A}$, so is $B_{j}$. Since $\mathscr{B}$ is infinite, after passing to its infinite subset, we can assume that $\left|b_{j+1}\right|_{\mathcal{A}}>t^{\prime}\left\|B_{j}\right\|+2 \Lambda_{1}$ for every $j \in \mathbb{N}$.

Suppose that there are indices $i<j$ such that $b_{i}$ is commensurable with $b_{j}$. Choose a shortest representative $b$ of the conjugacy class of $b_{i}$ in $G$; then $|b|_{\mathcal{A}} \leq$ $\left|b_{i}\right|_{\mathcal{A}}$. By the assumption, there exists $a \in G, k \in \mathbb{N}$ and $l \in \mathbb{Z} \backslash\{0\}$ such that $b^{l}=a b_{j}^{k} a^{-1}$.

Let $A$ and $B$ be shortest words over the alphabet $\mathcal{A}$ representing $a$ and $b$ respectively (note that the word $B$ is cyclically reduced in $G$ by construction). Then $B^{l}=A B_{j}^{k} A^{-1}$ in $G$, and one can use Lemma 10 to find words $U, V$ and $W$ such that $B_{j} \stackrel{G}{=} U V W$ where $\|U\|,\|W\| \leq \Lambda_{1} \leq \Lambda^{\prime}$ and $V$ is a $B$-periodic word. The latter equality implies that

$$
\|V\| \geq|V|_{\mathcal{A}} \geq\left|B_{j}\right|_{\mathcal{A}}-\|U\|-\|W\| \geq\left|b_{j}\right|_{\mathcal{A}}-2 \Lambda_{1}>t^{\prime}\left\|B_{i}\right\| \geq t^{\prime}\|B\|,
$$

which contradicts the $\left(\Lambda^{\prime}, t^{\prime}\right)$-aperiodicity of $b_{j}$. Therefore no two distinct elements of $\mathscr{B}$ can be commensurable and the statement is proved.

Lemma 12. Suppose that $G$ is a $\delta$-hyperbolic group, $0<\bar{\lambda} \leq 1, \bar{c} \geq 0$ and $\varkappa>0$. For any $\Lambda>0$ there exists $\Lambda^{\prime}>0$ such that for any $t^{\prime} \in \mathbb{N}$ there is $t \in \mathbb{N}$ satisfying the following.

Assume that $X_{1}, X_{2}$ are some words over $A$ representing elements of infinite order $x_{1}, x_{2} \in G$ and for each $i=1,2$, any path in $\Gamma(G, \mathcal{A})$ labelled by a power of $X_{i}$ is $(\bar{\lambda}, \bar{c})$-quasigeodesic. If $x_{1}$ is $\left(\Lambda^{\prime}, t^{\prime}\right)$-aperiodic, $\left\|X_{2}\right\| \leq \varkappa\left\|X_{1}\right\|$ and $x_{1}^{l}=a x_{2}^{k} a^{-1}$ for some $a \in G, k, l \in \mathbb{Z} \backslash\{0\}$, then $x_{2}$ is $(\Lambda, t)$-aperiodic.

Proof. Let $\lambda_{0}, c_{0}$ be from the claim of Lemma 9, $v_{0}=v_{0}\left(\delta, \lambda_{0}, c_{0}\right), \bar{v}=\bar{v}(\delta, \bar{\lambda}, \bar{c})$ be as in Lemma 1 and $\Lambda_{1}=2 \delta+2 \bar{v}$ be from Lemma 10. Set $\Lambda^{\prime}=4 \delta+3 \bar{v}+\Lambda_{1}+v_{0}+\Lambda$, 
choose an arbitrary $t^{\prime} \in \mathbb{N}$ and a positive integer $m$ so that

$$
m>\frac{1}{\bar{\lambda}}\left(\varkappa+2 \Lambda_{1}+\bar{c}\right)+2,
$$

and denote $t=m t^{\prime}$. Assume now that $x_{1}$ is $\left(\Lambda^{\prime}, t^{\prime}\right)$-aperiodic.

Arguing by contradiction, suppose that $x_{2}$ is not $(\Lambda, t)$-aperiodic. Then there is a geodesic path $p$ between 1 and $x_{2}$, points $a, b \in p$ and a $\left(\lambda_{0}, c_{0}\right)$-quasigeodesic path $r$ in $\Gamma(G, \mathcal{A})$ such that the label of $r$ is a $Z$-periodic word (for some cyclically reduced word $Z$ over $\mathcal{A}$ having infinite order in $G),\|r\| \geq t\|Z\|$ and $d\left(a, r_{-}\right) \leq \Lambda$, $d\left(b, r_{+}\right) \leq \Lambda$.

Using the assumptions together with Lemma 10 , one can find a $(\bar{\lambda}, \bar{c})$-quasigeodesic path $q$ in $\Gamma(G, \mathcal{A})$, where $\operatorname{lab}(q)$ is $X_{1}$-periodic and $d\left(p_{-}, q_{-}\right), d\left(p_{+}, q_{+}\right) \leq$ $\Lambda_{1}$.

Since the geodesic quadrilaterals in $\Gamma(G, \mathcal{A})$ are $2 \delta$-slim one obtains

$$
\begin{aligned}
a, b \in p & \subset \mathcal{N}_{2 \delta}\left(\left[p_{-}, q_{-}\right] \cup\left[q_{-}, q_{+}\right] \cup\left[q_{+}, p_{+}\right]\right) \\
& \subset \mathcal{N}_{2 \delta+\Lambda_{1}}\left(\left[q_{-}, q_{+}\right]\right) \subset \mathcal{N}_{2 \delta+\Lambda_{1}+\bar{v}}(q) .
\end{aligned}
$$

Thus, there are points $a^{\prime}, b^{\prime} \in q$ with $d\left(a, a^{\prime}\right), d\left(b, b^{\prime}\right) \leq 2 \delta+\Lambda_{1}+\bar{v}$; consequently $d\left(r_{-}, a^{\prime}\right), d\left(r_{+}, b^{\prime}\right) \leq 2 \delta+\Lambda_{1}+\bar{v}+\Lambda$. Using $2 \delta$-slimness of the geodesic quadrilateral with the vertices $r_{-}, r_{+}, a^{\prime}, b^{\prime}$ together with the property of quasigeodesics given by Lemma 1 , one gets

$$
\begin{aligned}
r & \subset \mathcal{N}_{v_{0}}\left(\left[r_{-}, r_{+}\right]\right) \subset \mathcal{N}_{v_{0}+2 \delta}\left(\left[r_{-}, a^{\prime}\right] \cup\left[a^{\prime}, b^{\prime}\right] \cup\left[b^{\prime}, r_{+}\right]\right) \\
& \subset \mathcal{N}_{v_{0}+2 \delta+2 \delta+\Lambda_{1}+\bar{v}+\Lambda}\left(\left[a^{\prime}, b^{\prime}\right]\right) \subset \mathcal{N}_{v_{0}+4 \delta+\Lambda_{1}+\bar{v}+\Lambda+\bar{v}}(q)=\mathcal{N}_{\Lambda^{\prime}-\bar{v}}(q) .
\end{aligned}
$$

Now let us estimate the length of $q$ using its quasigeodesity and the triangle inequality:

$$
\begin{aligned}
\|q\| & \leq \frac{1}{\lambda}\left(d\left(q_{-}, q_{+}\right)+\bar{c}\right) \\
& \leq \frac{1}{\bar{\lambda}}\left(d\left(1, x_{2}\right)+d\left(1, q_{-}\right)+d\left(q_{+}, x_{2}\right)+\bar{c}\right) \\
& \leq \frac{1}{\bar{\lambda}}\left(\left\|X_{2}\right\|+2 \Lambda_{1}+\bar{c}\right) \\
& \leq \frac{1}{\bar{\lambda}}\left(\varkappa\left\|X_{1}\right\|+2 \Lambda_{1}+\bar{c}\right) .
\end{aligned}
$$

Obviously, there is a path $\hat{q}$ in $\Gamma(G, \mathcal{A})$ such that $q$ is a subpath of $\hat{q}$, $\|\hat{q}\| \leq\|q\|+2\left\|X_{1}\right\|$ and $\operatorname{lab}(\hat{q}) \equiv X_{1}^{s}$ for some integer $s$. One has

$$
|s|\left\|X_{1}\right\|=\|\hat{q}\| \leq\|q\|+2\left\|X_{1}\right\| \text {, hence }|s| \leq \frac{1}{\lambda}\left(\varkappa+2 \Lambda_{1}+\bar{c}\right)+2<m
$$

due to the choice of $m$. Since $t=m t^{\prime}$, one can split $r$ in a concatenation of $m$ subpaths $r=r_{1} \ldots r_{m}$ such that $\operatorname{lab}\left(r_{j}\right)$ is a $Z$-periodic word and $\left\|r_{j}\right\| \geq t^{\prime}\|Z\|$ for all $j=1, \ldots, m$. The path $\hat{q}$, on the other hand, is a concatenation of $|s|$ subpaths 
$q=\hat{q}_{1} \ldots \hat{q}_{|s|}$, each of which is labelled by $X_{1}^{ \pm 1}$. Now, since $m>|s|$, one can use the pigeonhole principle to find $j, j^{\prime} \in\{1, \ldots, m\}, j \leq j^{\prime}$, and $k \in\{1, \ldots,|s|\}$, such that $\left(r_{j}\right)_{-},\left(r_{j^{\prime}}\right)_{+} \in \mathcal{N}_{\Lambda^{\prime}-\bar{v}}\left(\hat{q}_{k}\right) \subset \mathcal{N}_{\Lambda^{\prime}}\left(\left[\left(\hat{q}_{k}\right)_{-},\left(\hat{q}_{k}\right)_{+}\right]\right)$(here we utilize the $\left(\Lambda^{\prime}-\bar{v}\right)$-proximity of $r$ to $\hat{q}$ given by (3)). Let $r^{\prime}$ be the subpath of $r$ starting at $\left(r_{j}\right)_{-}$ and ending at $\left(r_{j^{\prime}}\right)_{+}$. Then lab $\left(r^{\prime}\right)$ is a $Z$-periodic word and $\left\|r^{\prime}\right\| \geq t^{\prime}\|Z\|$. But this contradicts the $\left(\Lambda^{\prime}, t^{\prime}\right)$-aperiodicity of $x_{1}$. The proof is finished.

Lemma 13. Let $G$ be a non-elementary hyperbolic group with $E(G)=\{1\}$. For every $\Lambda>0$ there is $t \in \mathbb{N}$ such that $G$ contains an infinite set $\left\{d_{i} \mid i \in \mathbb{N}\right\}$ of pairwise non-commensurable $(\Lambda, t)$-aperiodic elements of infinite order which are cyclically reduced and such that $E_{G}\left(d_{i}\right)=\left\langle d_{i}\right\rangle$ for every $i \in \mathbb{N}$.

Proof. Let $W_{1}, W_{2}, H, \lambda_{1}$ and $c_{1}$ be as in Lemma 8 , and let $\lambda_{0}, c_{0}$ be from Lemma 9 . Set $\bar{\lambda}=\min \left\{\lambda_{0}, \lambda_{1}\right\}, \bar{c}=\max \left\{c_{0}, c_{1}\right\}, \varkappa=1$ and find $\Lambda^{\prime}>0$ from the claim of Lemma 12. Denote $\Lambda^{\prime \prime}=\max \left\{\Lambda^{\prime}+v_{1}, \Lambda_{1}\right\}$, where $\Lambda_{1}$ and $v_{1}=v_{1}\left(\delta, \lambda_{1}, c_{1}\right)$ are given by Lemmas 11 and 1 .

According to Lemma 11 , there are $t^{\prime} \in \mathbb{N}$ and an infinite set $\mathcal{B}=\left\{b_{1}, b_{2}, \ldots\right\} \subset$ $F^{+}=\psi\left(F^{+}\left(W_{1}, W_{2}\right)\right) \subset H$ of pairwise non-commensurable $\left(\Lambda^{\prime \prime}, t^{\prime}\right)$-aperiodic elements of infinite order in $G$. Observe that by (1), for any $h \in H \backslash\{1\}$ the malnormality of $H$ implies that $E_{G}(h) \subset H$, hence $E_{G}(h)$ is cyclic as any torsionfree elementary group. In particular, for each $j \in \mathbb{N}$ there is $b_{j}^{\prime} \in F^{+}$such that $E_{G}\left(b_{j}\right)=\left\langle b_{j}^{\prime}\right\rangle$. For each $j \in \mathbb{N}$ one can choose a word $B_{j}^{\prime} \in F^{+}\left(W_{1}, W_{2}\right)$ representing $b_{j}^{\prime}$ such that any path in $\Gamma(G, \mathcal{A})$ labelled by a power of $B_{j}^{\prime}$ is $\left(\lambda_{1}, c_{1}\right)$ quasigeodesic (therefore it will also be $(\bar{\lambda}, \bar{c})$-quasigeodesic), because such a power will still belong to $F^{+}\left(W_{1}, W_{2}\right) \subset S\left(W_{1}, W_{2} ; 0\right)$. Now, since $b_{j}=\left(b_{j}^{\prime}\right)^{s}$ for some $s \in \mathbb{N}$, Lemma 1 immediately implies that $b_{j}^{\prime}$ is $\left(\Lambda^{\prime \prime}-v_{1}, t^{\prime}\right)$-aperiodic because $b_{j}$ is $\left(\Lambda^{\prime \prime}, t^{\prime}\right)$-aperiodic. Thus, the infinite set $\mathscr{B}^{\prime}=\left\{b_{1}^{\prime}, b_{2}^{\prime}, \ldots\right\}$ consists of $\left(\Lambda^{\prime}, t^{\prime}\right)$ aperiodic pairwise non-commensurable elements of infinite order in $G$, with the additional property that $E_{G}\left(b_{j}^{\prime}\right)=\left\langle b_{j}^{\prime}\right\rangle$.

For every $b_{j}^{\prime} \in \mathscr{B}^{\prime}$ choose a cyclically reduced element $d_{j} \in G$ and an element $a_{j} \in G$ such that $b_{j}^{\prime}=a_{j} d_{j} a_{j}^{-1}$. Note that the set $\mathscr{D}=\left\{d_{1}, d_{2}, \ldots\right\}$ is an infinite set of pairwise non-commensurable elements of infinite order in $G$, and that $E_{G}\left(d_{j}\right)=$ $a_{j}^{-1} E_{G}\left(b_{j}^{\prime}\right) a_{j}=\left\langle d_{j}\right\rangle$ is cyclic for every $j \in \mathbb{N}$.

Let $t$ be given by Lemma 12 . Choose a shortest word $D_{j}$ representing $d_{j}$ in $G$. Then $\left\|D_{j}\right\| \leq\left\|B_{j}\right\|=\varkappa\left\|B_{j}\right\|$ and so, according to Lemma 9 and the construction, one can apply Lemma 12 to show that $d_{j}$ is $(\Lambda, t)$-aperiodic for every $j \in \mathbb{N}$.

\section{Quotients of bounded exponent}

In this section we are going to prove Theorem 3. We will utilize the construction from [20] and explain how to obtain series of infinite quotients of a non-elementary 
hyperbolic group $G$ of bounded exponent by adding periodic relations $A^{n(i)}$ with different exponents $n(i)$.

Let $G$ be a non-elementary hyperbolic group. Fix a presentation $\left\langle\mathcal{A} \mid R \in \mathcal{R}_{0}\right\rangle$ of $G$ as in the beginning of Section 5. Since $E(G)$ is the maximal finite normal subgroup of $G$, the quotient $\hat{G}=G / E(G)$ is again a non-elementary hyperbolic group with the additional property $E(\hat{G})=\{1\}$. After replacing $G$ with $\hat{G}$, we will further assume that $E(G)=\{1\}$.

Let $v_{0}=v_{0}\left(\lambda_{0}, c_{0}\right)$ be the constant provided by Lemma 1 , where the pair $\left(\lambda_{0}, c_{0}\right)$ is chosen according to Lemma 9. Choose the auxiliary parameters (such as $\Lambda, \alpha$, $\beta, \gamma, \varepsilon, \rho, \theta, n$, etc.) as in $\S 3$ of [20]. We refer to [20], [19] for the values and estimates of the parameters; in the present section we will explicitly use the following inequalities: $\theta>\alpha+3 \beta, 2 \gamma+1 / 2<\rho$. The choice of the parameter $n$, which is a very large integer, is made after all the other parameters are chosen. Increasing $n$ (that is, multiplying it by a power of 2) if necessary, and using Lemma 13, we find an infinite set $\mathcal{B}=\left\{B_{1}, B_{2}, \ldots\right\}$ of cyclically reduced words satisfying the following properties:

1. Elements of the group $G$ represented by words $B_{1}, B_{2}, \ldots$ are pairwise noncommensurable elements of infinite order that generate their respective (cyclic) elementary subgroups $E_{G}\left(B_{k}\right), k=1,2, \ldots$

2. For every $B \in \mathscr{B}$, the word $B^{ \pm 3}$ represents a $\left(\Lambda+v_{0}, \beta n\right)$-aperiodic element.

Passing to a subsequence, we can assume that $\left\|B_{1}\right\|>n^{2}$ and $\left\|B_{k+1}\right\|>n^{2}\left\|B_{k}\right\|$ for $k \geq 1$. Given a sequence $\omega=\left(\omega_{k}\right)_{k=1}^{\infty}$ of 0 's and 1's, we are going to construct a periodic quotient $G_{\omega}(\infty)$ of $G$ of exponent $2 n$.

We set $G_{\omega}(0)=G$, introduce a total order $\prec$ and define simple words as in Section 5. Assuming that the presentation of $G_{\omega}(i-1)$ has already been constructed, choose the period $A_{i}$ (of rank $i$ ) as in Section 5 .

We distinguish the following two cases:

(1) A non-trivial power of some word $B_{k} \in \mathcal{B}$ is conjugate in $G_{\omega}(i-1)$ to an element $A_{i}^{\ell} F$, for some $k \geq 1, \ell \in \mathbb{Z}, \ell \neq 0, F \in \mathcal{F}\left(A_{i}\right)$. (It follows from Lemma 11, Lemma 16 below and Lemma 18.5 (c) [19], [20] in rank $i-1$ that such $k$ is unique.) In this case we shall say that the period $A_{i}$ is special.

(2) None of non-trivial powers of words from $\mathcal{B}$ is conjugate in $G_{\omega}(i-1)$ to an element of the form $A_{i}^{\ell} F$, for some $\ell \in \mathbb{Z}, \ell \neq 0, F \in \mathcal{F}\left(A_{i}\right)$.

Set $n(i)=n$ if case (1) holds and $\omega_{k}=0$. Otherwise set $n(i)=2 n$.

The group $G_{\omega}(i)$ is obtained by imposing on $G_{\omega}(i-1)$ the relation $A_{i}^{n(i)}=1$ :

$$
G_{\omega}(i)=\left\langle\mathcal{A} \mid R \in \mathcal{R}_{0} \cup\left\{A_{1}^{n(1)}, A_{2}^{n(2)}, \ldots, A_{i}^{n(i)}\right\}\right\rangle .
$$

Analysis of groups $G_{\omega}(i)$ is done using geometric interpretation of deducing consequences from defining relations according to the scheme of [20] (and [19]). We refer the reader to [20], [19] for the definitions of a bond, a contiguity subdiagram 
and its standard contour, a degree of contiguity, a reduced diagram, (strict) rank and type of a diagram, a simple word in rank $i$ (some of these concepts were defined in Section 4).

References to lemmas from [20], as well as lemmas from [19] and their analogs in [20], will be made preserving the numeration of [20] and of [19].

We note that our modification of the construction implies obvious changes in formulations and proofs from [20], [19]: a cell of rank $i$ now corresponds to the relation $A_{i}^{n(i)}=1$ and $n(i)$ appears instead of $n$ in all the estimates of the length of its boundary; the order of the period $A_{i}$ in $G_{\omega}(\infty)$ is now $n(i)$ instead of $n$; finite subgroups of $G_{\omega}(i)$ are isomorphically embedded into a direct product of a direct power of the dihedral group $D(4 n)$ (instead of $D(2 n)$ ) and a group elementary associated with $G$ (see [20]); in the inductive step from $G_{\omega}(i)$ to $G_{\omega}(i+1)$ (in arguments from [19], $\S \S 18,19$, and their analogues from [20]) $n$ is replaced by $n(i+1)$. The crucial part that needs explanation is the validity of equations in Lemma 15.10 [19], [20].

The following lemma about structure of diagrams precedes Lemma 3.1 [19], [20].

Lemma 14. Let $B \in \mathscr{B}$ and let $\Gamma$ be a contiguity subdiagram of a cell $\Pi$ to a $B$-periodic section $p$ of the contour of a reduced diagram $\Delta$ of rank $i$ such that $\operatorname{lab}(\Gamma \wedge p)$ is a subword of $B^{ \pm 3}$. Then $r(\Gamma)=0$ and the contiguity degree of $\Pi$ to $p$ via $\Gamma$ is less than $\beta$.

Proof. We prove this lemma by contradiction. Assume that the triple $(p, \Gamma, \Pi)$ is a counterexample, where the contiguity subdiagram $\Gamma$ has a minimal type. Let the standard contour of $\Gamma$ be $\partial \Gamma=d_{1} p_{1} d_{2} q$, where $p_{1}=\Gamma \wedge p, q=\Gamma \wedge \Pi$. The bonds defining $\Gamma$ are 0 -bonds (otherwise $(p, \Gamma, \Pi)$ is not minimal). This means that $\max \left\{\left\|d_{1}\right\|,\left\|d_{2}\right\|\right\}<\Lambda$. Assuming that $r(\Gamma)>0$, by Lemma 5.7 [19], [20] $(\tau(\Gamma)<\tau(\Delta))$ there is a $\theta$-cell. However, by minimality of $(p, \Gamma, \Pi)$ the degree of contiguity of any cell from $\Gamma$ to $p_{1}$ is less than $\beta$ and the contiguity degree of any cell from $\Gamma$ to $q$ is less than $\alpha$ by Lemma 3.4 [19], [20] (again, $\tau(\Gamma)<\tau(\Delta)$ ). Contiguity degrees of cells of positive ranks to sections $d_{1}$ and $d_{2}$ of $\partial \Gamma$ are bounded from above by $\beta$ in view of inequality $\max \left\{\left\|d_{1}\right\|,\left\|d_{2}\right\|\right\}<\Lambda$ and Lemma 6.1 [19], [20]. The fact that $\theta>\alpha+3 \beta$ implies that $\Gamma$ does not have cells of positive ranks, i.e., $\Gamma$ is a diagram over the presentation of $G$. Note that the endpoints of the path $p_{1}$ are within $v_{0}$ from the geodesic (in the Cayley graph of $G$ ) segment that connects the endpoints of $p$. Assuming that the contiguity degree of $\Pi$ to $p$ via $\Gamma$ is greater than or equal to $\beta$, we arrive at a contradiction with $\left(\Lambda+v_{0}, \beta n\right)$-aperiodicity of $B^{3}$ in $G$. The lemma is proved.

The formulation of the analogue of Lemma 15.10 [19], [20] is changed as follows. For any finite subgroup $\mathcal{E}$ of $G_{\omega}(i)$ equations from [19], [20] hold with the exponent $n$ replaced by $2 n$. If, in addition, $\mathcal{E}$ is a finite subgroup of $G_{\omega}(i)$, conjugate to a subgroup of $\mathcal{K}\left(A_{j}\right), j \leq i$, where the period $A_{j}$ is special, then the equations from 
part (a) of Lemma 15.10 [19], [20] hold with exponent $n$. The proof is retained. In the case the relation of rank $j$ is $A_{j}^{n}=1$, the claim follows from Lemmas 17 and 18 (below) applied in smaller rank: the subgroup $\mathcal{F}\left(A_{j}\right)$ is trivial, there are no $\mathscr{F}\left(A_{j}\right)$-involutions, and so the equations are trivially satisfied.

The inductive step from rank $i$ to $i+1$ starts with the following lemmas.

Lemma 15. If a word $B$ from $\mathcal{B}$ is of infinite order in rank $i$, then $B$ is simple in rank $i$.

Proof. By the choice of the set $\mathcal{B}$ and the definition of simple words, $B$ is simple in rank 0 and therefore it is simple in all ranks $i \leq i_{0}$ (as in [20], $i_{0}$ stands for the maximal rank for which $\left\|A_{i_{0}}\right\|<C$ ). Let now $i>i_{0}$. Assume that $B$ is not simple in rank $i$. By definition of a simple in rank $i$ word, and because $B$ has infinite order in rank $i$, this means that $B$ is not cyclically reduced in rank $i$, that is, there exists a word $X$ conjugated to $B$ in rank $i$ such that $\|X\|<\|B\|$. Let $\Delta$ be an annular reduced diagram representing conjugacy of $B$ and $X$ in rank $i$. Denote by $p, q$ contours of $\partial \Delta$ labelled by $B, X$, respectively. Without loss of generality we may assume that the path $q$ is cyclically geodesic in $\Delta$, i.e., geodesic in its homotopy class in $\Delta$. If $r(\Delta)>0$ then, by Lemma 5.7 [19], [20], there is a cell $\Pi$ of positive rank in $\Delta$ and contiguity subdiagrams $\Gamma_{p}, \Gamma_{q}$ of $\Pi$ to $p, q$ respectively such that the sum of contiguity degrees of $\Pi$ to $p, q$ via $\Gamma_{p}, \Gamma_{q}$ is greater than $\theta$. However, by Lemma 14, $\left\|\Gamma_{p} \wedge \Pi\right\|<\beta\|\partial \Pi\|$ and, by Lemma 3.3 [19], [20], $\left\|\Gamma_{q} \wedge \Pi\right\|<\alpha\|\partial \Pi\|$. Thus, the inequality $\theta>\alpha+\beta$ implies that $\Delta$ does not have cells of positive rank. But this is a contradiction since the word $B$ was chosen to be cyclically reduced in $G=G(0)$.

Lemma 16. Let $l \neq k$ and assume that some words $B_{l}, B_{k} \in \mathcal{B}$ are of infinite order in the group $G_{\omega}(i)$. Then $B_{l}$ and $B_{k}$ are not commensurable in $G_{\omega}(i)$.

Proof. Let $l>k$. By the choice of the set $\mathcal{B}$, the words $B_{l}$ and $B_{k}$ are not commensurable in $G=G_{\omega}(0)$. Let now $i \geq 1$, and assume, on the contrary, that the words $B_{l}, B_{k} \in \mathscr{B}$ have infinite order and are commensurable in $G_{\omega}(i)$. Let $\Delta$ be an annular reduced diagram of rank $i$ for the conjugacy of some non-trivial powers $B_{l}^{s}$ and $B_{k}^{t}$. Denote by $p$ and $q$ contours of $\Delta$ so that $\operatorname{lab}(p) \equiv B_{l}^{s}, \operatorname{lab}(q) \equiv B_{k}^{t}$. The words $B_{l}$, $B_{k}$ are not commensurable in $G_{\omega}(0)$, hence $r(\Delta)>0$. By Lemma 5.7 [19], [20], there is a cell $\Pi$ of positive rank $j \leq i$ and contiguity subdiagrams $\Gamma_{p}, \Gamma_{q}$ of $\pi$ to $p, q$ respectively, such that $\left\|\Gamma_{p} \wedge \Pi\right\|+\left\|\Gamma_{q} \wedge \Pi\right\|>\theta\|\partial \Pi\|$.

Note that $\left\|A_{j}\right\| \leq\left\|B_{k}\right\|<n^{-2}\left\|B_{l}\right\|$. Therefore $\|\partial \Pi\|<\left\|B_{l}\right\|$ and $\operatorname{lab}\left(\Gamma_{p} \wedge p\right)$ is a subword of $B_{l}^{ \pm 3}$. It follows from Lemma 14 that $\left\|\Gamma_{p} \wedge \Pi\right\|<\beta\|\partial \Pi\|$. Вy Lemmas 15 and 3.4 [19], [20], $\left\|\Gamma_{q} \wedge \Pi\right\|<\alpha\|\partial \Pi\|$. This is a contradiction since $\theta>\alpha+\beta$.

The following lemmas describe the structure of finite subgroups associated to the periods distinguished by the elements from the set $\mathcal{B}$. They precede Lemma 18.5 [19],[20]. 
Lemma 17. Let a non-trivial power $B^{r}$ of some word $B \in \mathscr{B}$ be conjugate in $G_{\omega}(i)$ to an element $A_{i+1}^{\ell} F$, for some $\ell \in \mathbb{Z}, \ell \neq 0, F \in \mathcal{F}\left(A_{i+1}\right)$. Then $\mathcal{F}\left(A_{i+1}\right)=\{1\}$.

Proof. The word $B$ is of infinite order in rank $i$ and by Lemma 15 it is simple in rank $i$. Let $K$ be a finite subgroup of $G_{\omega}(i)$ normalized by $B^{r}$. Our aim is to show that $K$ is trivial. Assuming the contrary, choose a word $F_{0}$ representing a non-trivial element from $K$. Since $K$ is finite and is normalized by $B^{r}$, there exists an integer $s>0$ such that

$$
B^{-s} F_{0} B^{s} \stackrel{i}{=} F_{0} .
$$

Choose $s$ such that $|s|>n^{2}\left\|F_{0}\right\|$ and let $\Delta$ be a disk reduced diagram of rank $i$ representing this equation with the standard contour $\partial \Delta=b p c q$, where lab $(b) \equiv F_{0}$, $\operatorname{lab}(p) \equiv B^{s}, \operatorname{lab}(c) \equiv F_{0}^{-1}, \operatorname{lab}(q) \equiv B^{-s}$. Assuming $r(\Delta)>0$, by Lemma 5.7 [19], [20], one will find a $\theta$-cell $\Pi$ in $\Delta$. Denote $j=r(\Pi)$ (observe that $\|B\| \geq\left\|A_{j}\right\|$ ) and let $\Gamma_{b}, \Gamma_{p}, \Gamma_{c}$ and $\Gamma_{q}$ be contiguity subdiagrams (some of them may be absent) of $\Pi$ to $b, p, c$ and $q$ respectively. Sections $b$ and $c$ may be regarded as geodesic sections of $\partial \Delta$. Therefore, by Lemma 3.3 [19], [20],

$$
\max \left\{\left\|\Gamma_{b} \wedge \Pi\right\|,\left\|\Gamma_{c} \wedge \Pi\right\|\right\}<\alpha\|\partial \Pi\| .
$$

Suppose that $\left\|\Gamma_{b} \wedge \Pi\right\|+\left\|\Gamma_{c} \wedge \Pi\right\|>(\alpha+\beta)\|\partial \Pi\|$. In particular, both $\Gamma_{b}$ and $\Gamma_{c}$ are present, and the contiguity degrees of $\Pi$ to $b$ and to $c$ via $\Gamma_{b}$ and $\Gamma_{c}$ are greater than $\beta$. Denote the standard contours $\partial \Gamma_{b}=u_{1} b_{1} u_{2} u_{b}$ and $\partial \Gamma_{c}=v_{1} c_{1} v_{2} u_{c}$, where $b_{1}=\Gamma_{b} \wedge b, u_{b}=\Gamma_{b} \wedge \Pi, c_{1}=\Gamma_{c} \wedge c, u_{c}=\Gamma_{c} \wedge \Pi$. By Lemma 3.1 [19], [20],

$$
\max \left\{\left\|u_{1}\right\|,\left\|u_{2}\right\|,\left\|v_{1}\right\|,\left\|v_{2}\right\|\right\}<\gamma\|\partial \Pi\| .
$$

It follows that initial and terminal vertices $p_{-}$and $p_{+}$of the path $p$ can be joined in $\Delta$ by a path of length less than

$$
\left\|F_{0}\right\|+\gamma\|\partial \Pi\|+1 / 2\|\partial \Pi\|+\gamma\|\partial \Pi\|+\left\|F_{0}\right\|=2\left\|F_{0}\right\|+(2 \gamma+1 / 2) n\left\|A_{j}\right\|<\rho\|p\| .
$$

This contradicts Lemma 6.1 [19], [20] since $p$ is a smooth section of $\partial \Delta$ and of the boundary of any subdiagram of $\Delta$ that contains $p$ in its contour. Consequently, $\left\|\Gamma_{b} \wedge \Pi\right\|+\left\|\Gamma_{c} \wedge \Pi\right\| \leq(\alpha+\beta)\|\partial \Pi\|$.

Let us now assume that $\Gamma_{p}$ is present. Denote the standard contour $\partial \Gamma_{p}=$ $d_{1} p_{1} d_{2} p_{2}$, where $p_{1}=\Gamma_{p} \wedge p$ and $p_{2}=\Gamma_{p} \wedge \Pi$. If $\left\|p_{2}\right\| \geq \beta\|\partial \Pi\|$, then by Lemma 15 and Lemma 20.2 [19], [20] applied to $\Gamma_{p}\left(\tau\left(\Gamma_{p}\right)<\tau(\Delta)\right)$, one gets $\left\|p_{1}\right\|<(1+\varepsilon)\|B\|$. Hence, $\operatorname{lab}\left(p_{1}\right)$ is a subword of $B^{ \pm 3}$, and Lemma 14 yields a contradiction. Therefore, $\left\|\Gamma_{p} \wedge \Pi\right\|<\beta\|\partial \Pi\|$. Arguing in the same way, we obtain that $\left\|\Gamma_{q} \wedge \Pi\right\|<\beta\|\partial \Pi\|$ in the case $\Gamma_{q}$ is present. It follows that

$$
\left\|\Gamma_{b} \wedge \Pi\right\|+\left\|\Gamma_{p} \wedge \Pi\right\|+\left\|\Gamma_{c} \wedge \Pi\right\|+\left\|\Gamma_{q} \wedge \Pi\right\|<(\alpha+3 \beta)\|\partial \Pi\|<\theta\|\partial \Pi\|,
$$

contradicting the definition of a $\theta$-cell.

Therefore, $r(\Delta)=0$ and equality $B^{-s} F_{0} B^{s}=F_{0}$ holds in the group $G(0)$. This means that $F_{0}$ belongs to the elementary subgroup of $B$ in $G(0)$. Since the elementary subgroup of $B$ is cyclic, $F_{0}$ is trivial in $G_{\omega}(i)$. Consequently, $\mathscr{F}\left(A_{i+1}\right)=\{1\}$. 
Lemma 18. Let a non-trivial power $B^{r}$ of some word $B \in \mathscr{B}$ be conjugate in $G_{\omega}(i)$ to an element $A_{i+1}^{\ell} F$, for some $\ell \in \mathbb{Z}, \ell \neq 0, F \in \mathcal{F}\left(A_{i+1}\right)$. Then there are no $\mathcal{F}\left(A_{i+1}\right)$-involutions.

Proof. By Lemma 17, we may assume that $B^{r} \stackrel{i}{=} X^{-1} A_{i+1}^{\ell} X$ for some word $X$. Let $J$ be a $\mathscr{F}\left(A_{i+1}\right)$-involution. Then $J_{0}^{-1} B^{r} J_{0} \stackrel{i}{=} B^{-r}$, where $J_{0} \stackrel{i}{=} X^{-1} J X$. It follows from Lemma 17 and the definition of a $\mathcal{F}\left(A_{i+1}\right)$-involution that $J$ (and therefore $J_{0}$ ) is of order two in rank $i$.

Choose $s$ such that $|s|>n^{2}\left\|J_{0}\right\|$ and consider a reduced diagram $\Delta$ of rank $i$ representing the equation

$$
J_{0}^{-1} B^{s} J_{0} \stackrel{i}{=} B^{-s} .
$$

Arguing as in the proof of Lemma 17, we obtain that $r(\Delta)=0$. Consequently, $J_{0}^{-1} B^{s} J_{0} \stackrel{0}{=} B^{-s}$, and $J_{0}$ belongs to the elementary subgroup of $B$ in $G(0)$. Since the order of $J_{0}$ in $G_{\omega}(i)$ is finite while the order of $B$ in $G_{\omega}(i)$ is infinite, it follows that $J_{0} \stackrel{i}{=} 1$ (and therefore $J \stackrel{i}{=} 1$ ).

The inductive proof is completed as in [19], [20]. As a result, we obtain a presentation

$$
G_{\omega}(\infty)=\left\langle\mathcal{A} \mid R \in \mathcal{R}_{0} \cup\left\{A_{1}^{n(1)}, A_{2}^{n(2)}, \ldots\right\}\right\rangle .
$$

of an infinite group of exponent $2 n$. The proof that $G_{\omega}(\infty)$ has trivial center repeats the argument from $\$ 21$ of [19].

Now we explain that there is a continuum of pairwise non-isomorphic groups among $\left\{G_{\omega}(\infty)\right\}_{\omega}$. We notice first that, by Lemmas 10.1 and 10.2 ([20], [19]), and Lemma 16, the set of special periods is infinite and there is a special period corresponding to each element $B \in \mathcal{B}$. Presentations of groups $G_{\omega}(\infty)$ are now distinguished by the orders of special periods: if $\omega$ and $\omega^{\prime}$ are two different sequences of 0 's and 1's, find the first place (say, $k$ ) where they differ. By construction and Lemma 10.4 ([20], [19]), the special period that corresponds to $B_{k}$ has different orders in $G_{\omega}(\infty)$ and $G_{\omega^{\prime}}(\infty)$, meaning that $G_{\omega}(\infty)$ and $G_{\omega^{\prime}}(\infty)$ are quotients of $G$ over different normal subgroups. By Remark 2, we see that there is a continuous family of pairwise non-isomorphic infinite centerless quotients of $G$ of exponent $2 n$. Theorem 3 is proved.

\section{Periodic quotients of large groups}

This section is devoted to proving Theorem 4 and Corollaries 1,2 .

Let $\mathcal{C}$ be a collection of groups. A group $G$ is said to be residually in $\mathcal{C}$ if for every $g \in G \backslash\{1\}$ there is a homomorphism $\varphi: G \rightarrow H$ for some group $H \in \mathcal{C}$ such that $\varphi(g) \neq 1$. For a prime number $p$, denote by $\ell_{p}$ the collection consisting of all finite $p$-groups. Since every finite $p$-group is nilpotent we can make 
Remark 3. 1) If $H$ is a finite $p$-group, then there is an integer $n \in \mathbb{N}$ such that $\delta_{n}^{p}(H)=\{1\}$.

2) For a finitely generated group $G, \bigcap_{j=0}^{\infty} \delta_{j}^{p}(G)=\{1\}$ if and only if $G$ is residually in $\ell_{p}$.

Since free groups are residually in $\ell_{p}$ for any prime $p$ ([23], 14.2.2), we have

Lemma 19. If $F$ is a free group and $p \in \mathbb{N}$ is a prime number, then one has $\bigcap_{j=0}^{\infty} \delta_{j}^{p}(F)=\{1\}$.

Throughout this section $F_{2}$ will denote the free group of rank 2. For a group $G$, we will write $G \rightarrow F_{2}$ if there exists an epimorphism from $G$ to $F_{2}$. The lemma below was proved by Olshanskii and Osin in [37]. (We apply it in the special case when $\Pi=(p, p, \ldots)$ is a constant sequence. $)$

Lemma 20 ([37], Lemma 3.2). Let $G$ be a finitely generated group, $N \triangleleft G$ be a normal subgroup of finite index and let $p \in \mathbb{N}$ be a prime number. Suppose that $N \rightarrow F_{2}$. Then for any element $g \in N$ there is $m>0$ such that if $g^{n} \in \delta_{m}^{p}(N)$, then $\delta_{m}^{p}(N) /\left\langle\left\langle g^{n}\right\rangle\right\rangle^{G} \rightarrow F_{2}$.

(Here we modified the original formulation of [37], Lemma 3.2, by replacing $\delta_{r}^{p}(G)$ with an arbitrary finite index normal subgroup $N$, observing that the proof continues to be valid in this more general situation).

Lemma 21. Suppose that $G$ is a finitely generated group, $N \triangleleft G$ is a finite index normal subgroup, such that $N \rightarrow F_{2}$, and $p$ is an arbitrary prime number. Then for each sequence $\omega \in \Omega=\{0,1\}^{\mathbb{N}}$, there is a group $H_{\omega}$ containing a normal subgroup $Q_{\omega} \triangleleft H_{\omega}$ such that

(i) $H_{\omega}$ is a quotient of $G$;

(ii) $H_{\omega} / Q_{\omega} \cong G / N$;

(iii) $Q_{\omega}$ is a periodic p-group;

(iv) $\bigcap_{i=0}^{\infty} \delta_{i}^{p}\left(Q_{\omega}\right)=\{1\}$;

(v) if $\omega \neq \omega^{\prime} \in \Omega$ then there is $v \in \mathbb{N}$ such that $\left|Q_{\omega} / \delta_{v}^{p}\left(Q_{\omega}\right)\right| \neq\left|Q_{\omega^{\prime}} / \delta_{v}^{p}\left(Q_{\omega^{\prime}}\right)\right|$; consequently $Q_{\omega} \neq Q_{\omega^{\prime}}$.

Proof. Our argument will be similar to the one used in the proof of [37], Thm. 1.2.

First, enumerate all the elements of $N: N=\left\{f_{1}, f_{2}, \ldots\right\}$. Let $\Omega_{i}=\{0,1\}^{i}$ be the set of sequences of 0's and 1's of length $i, i \in \mathbb{N}$, and let $\Omega_{0}$ consist of the empty sequence $\emptyset$. Denote $G_{\emptyset}=G, r_{\emptyset}=q_{\emptyset}=0$, and assume that for some $i \geq 0$ and for every $\iota \in \Omega_{i}$ we have already constructed the quotient group $G_{\iota}$ of $G$ and integers $r_{\iota}, q_{\iota} \geq 0$ such that the images of $f_{1}, \ldots, f_{i}$ have finite orders in $G_{\iota}$ and $\delta_{r_{\iota}}^{p}\left(N_{\iota}\right) \rightarrow F_{2}$, where $N_{\iota}$ is the image of $N$ in $G_{\iota}$ under the natural homomorphism (the numbers $q_{\iota}$ are auxiliary, and will be used during the inductive argument). 
Choose any $\zeta=\left(\zeta_{1}, \ldots, \zeta_{i+1}\right) \in \Omega_{i+1}$ and take $\iota=\left(\zeta_{1}, \ldots, \zeta_{i}\right) \in \Omega_{i}$.

Observe that the subgroup $E_{\iota}=\bigcap_{j=0}^{\infty} \delta_{j}^{p}\left(N_{\iota}\right)$ is characteristic in $N_{\iota}$, hence it is normal in $G_{\iota}$. Denote $K_{\iota}=G_{\iota} / E_{\iota}, L_{\iota}=N_{\iota} / E_{\iota} \triangleleft K_{\iota}$; then

$$
\bigcap_{j=0}^{\infty} \delta_{j}^{p}\left(L_{\iota}\right)=\{1\}
$$

Since $E_{\iota}=\bigcap_{j=0}^{\infty} \delta_{j}^{p}\left(\delta_{r_{\iota}}^{p}\left(N_{\iota}\right)\right)$, Lemma 19 implies that $\delta_{r_{\iota}}^{p}\left(L_{\iota}\right) \rightarrow F_{2}$. Let $l \geq i+1$ be the smallest index such that the image of $f_{l}$ has infinite order in $K_{l}$. Denote by $g$ the image of $\left(f_{l}\right)^{p^{r_{l}}}$ in $K_{l}$; thus $g \in \delta_{r_{l}}^{p}\left(L_{l}\right)$ is an element of infinite order.

Now we apply Lemma 20 to find $m \in \mathbb{N}$ such that

$$
\delta_{r_{\iota}+m}^{p}\left(L_{\iota}\right) /\left\langle\left\langle g^{n}\right\rangle\right\rangle^{K_{\iota}}=\delta_{m}^{p}\left(\delta_{r_{\iota}}^{p}\left(L_{\iota}\right)\right) /\left\langle\left\langle g^{n}\right\rangle\right\rangle^{K_{\iota}} \rightarrow F_{2}
$$

for any integer $n$ such that $g^{n} \in \delta_{r_{l}+m}^{p}\left(L_{\iota}\right)$.

Set $s=\max \left\{r_{\iota}+m, q_{\iota}\right\}$ so that $g^{p^{s}} \in \delta_{s}^{p}\left(L_{\iota}\right) \leq \delta_{r_{l}+m}^{p}\left(L_{\iota}\right) \cap \delta_{q_{\iota}}^{p}\left(L_{\iota}\right)$. By (4) one can find the smallest integer $v_{\iota}$ such that $g^{p^{s}} \notin \delta_{v_{\iota}}^{p}\left(L_{\iota}\right)$. Set $r_{\zeta}=r_{\iota}+m$ and $q_{\zeta}=v_{\iota}$. Note that $q_{\zeta}>s \geq q_{\iota}$, and the integers $r_{\zeta}$ and $q_{\zeta}$ only depend on the prefix $\iota$ of the sequence $\zeta$ and are independent of the value of $\zeta_{i+1}$.

If $\zeta_{i+1}=0$, define $n_{\zeta}=p^{s}$ (thus, $g^{n_{\zeta}} \in L_{\iota} \backslash \delta_{v_{\iota}}^{p}\left(L_{\iota}\right)$ ), otherwise, if $\zeta_{i+1}=1$, define $n_{\zeta}=p^{q_{\zeta}}$ (thus, $g^{n_{\zeta}} \in \delta_{v_{\iota}}^{p}\left(L_{\iota}\right)$ ). Denote $G_{\zeta}=K_{\iota} /\left\langle\left\langle g^{n_{\zeta}}\right\rangle\right\rangle^{K_{\iota}}$ and let $N_{\zeta}$ be the image of $L_{\iota}$ in $G_{\zeta}$ (see the diagram below).

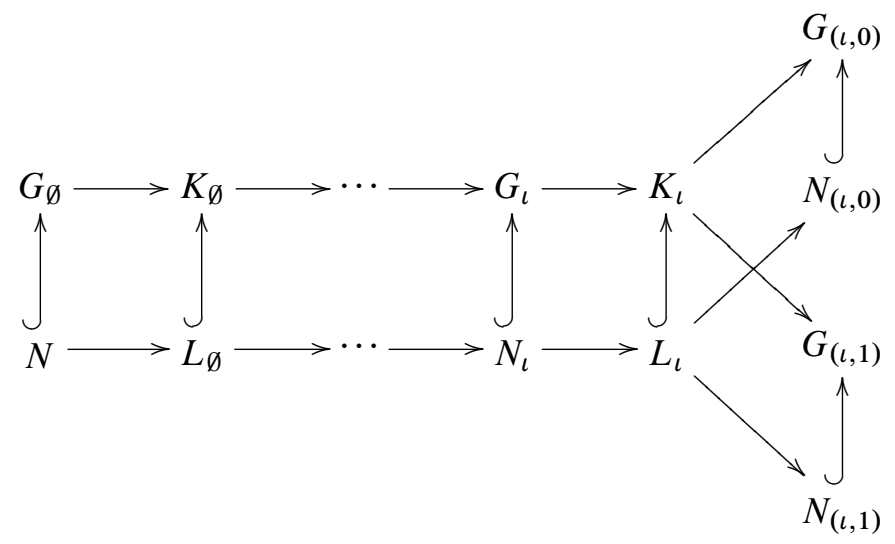

Observe that

$$
\delta_{r_{\zeta}}^{p}\left(N_{\zeta}\right) \rightarrow F_{2}
$$

by (5),

$$
N_{\zeta} / \delta_{u}^{p}\left(N_{\zeta}\right) \cong L_{\iota} / \delta_{u}^{p}\left(L_{\iota}\right) \cong N_{\iota} / \delta_{u}^{p}\left(N_{\iota}\right)
$$

for every $u \leq q_{\iota}$, and

$$
\left|N_{(\iota, 0)} / \delta_{v_{\iota}}^{p}\left(N_{(\iota, 0)}\right)\right|<\left|N_{(\iota, 1)} / \delta_{v_{\iota}}^{p}\left(N_{(\iota, 1)}\right)\right|=\left|L_{\iota} / \delta_{v_{\iota}}^{p}\left(L_{\iota}\right)\right|=\left|N_{\iota} / \delta_{v_{\iota}}^{p}\left(N_{\iota}\right)\right|<\infty .
$$


Thus, for each $i \geq 0$ and each $\iota \in \Omega_{i}$, we have constructed a quotient $G_{\iota}$ of $G$. Additionally, we have an epimorphism $G_{\iota} \rightarrow G_{\zeta}$ whenever $\iota$ is a prefix of $\zeta$. Therefore, for every $\omega \in \Omega$ we can define the group $G_{\omega}$ as a direct limit of the groups $G_{\iota}$, where $\iota$ runs over all prefixes of $\omega$. Let $N_{\omega} \triangleleft G_{\omega}$ be the image of $N$ in $G_{\omega}$, and let $H_{\omega}$ be the quotient of $G_{\omega}$ by $E_{\omega}=\bigcap_{j=0}^{\infty} \delta_{j}^{p}\left(N_{\omega}\right)$. Finally, let $Q_{\omega}$ be the image of $N_{\omega}$ in $H_{\omega}$.

By construction, $Q_{\omega}$ is periodic (as a quotient of $N$ ) and $\bigcap_{j=0}^{\infty} \delta_{j}^{p}\left(Q_{\omega}\right)=\{1\}$. Since $N$ is finitely generated, so is $Q_{\omega}$. Therefore, for every $j, Q_{\omega} / \delta_{j}^{p}\left(Q_{\omega}\right)$ is a finite group whose order divides $p^{j}$, hence $Q_{\omega}$ is a $p$-group.

The second claim of the lemma also follows from the construction. It remains for us to prove the fifth claim. Suppose that $\omega \neq \omega^{\prime} \in \Omega$, and let $\iota \in \Omega_{i}, i \geq 0$, be the longest common prefix of $\omega$ and $\omega^{\prime}$.

By (6) we have

$$
Q_{\omega} / \delta_{v_{\iota}}^{p}\left(Q_{\omega}\right) \cong N_{\omega} / \delta_{v_{\iota}}^{p}\left(N_{\omega}\right) \cong N_{\zeta} / \delta_{v_{\iota}}^{p}\left(N_{\zeta}\right)
$$

and

$$
Q_{\omega^{\prime}} / \delta_{v_{\iota}}^{p}\left(Q_{\omega^{\prime}}\right) \cong N_{\omega^{\prime}} / \delta_{v_{\iota}}^{p}\left(N_{\omega^{\prime}}\right) \cong N_{\zeta^{\prime}} / \delta_{v_{\iota}}^{p}\left(N_{\zeta^{\prime}}\right),
$$

where $\zeta, \zeta^{\prime} \in \Omega_{i+1}$ are prefixes of length $i+1$ of $\omega$ and $\omega^{\prime}$, respectively. Using this together with (7) we obtain that

$$
\left|Q_{\omega} / \delta_{v_{\iota}}^{p}\left(Q_{\omega}\right)\right|=\left|N_{\zeta} / \delta_{v_{\iota}}^{p}\left(N_{\zeta}\right)\right| \neq\left|N_{\zeta^{\prime}} / \delta_{v_{\iota}}^{p}\left(N_{\zeta^{\prime}}\right)\right|=\left|Q_{\omega^{\prime}} / \delta_{v_{\iota}}^{p}\left(Q_{\omega^{\prime}}\right)\right|,
$$

yielding (v). Thus the lemma is proved.

Proof of Theorem 4. Applying Lemma 21 to $G, N$ and $p$, for each $\omega \in \Omega$ we obtain a group $H_{\omega}$ together with a normal subgroup $Q_{\omega}$ of index $k=|G: N|$ enjoying the properties (i)-(v) from the claim of Lemma 21.

Since $Q_{\omega}$ is finitely generated, property (iv) implies that it is residually finite. And since $\left|H_{\omega}: Q_{\omega}\right|=|G: N|<\infty$ by (ii), $H_{\omega}$ is residually finite for every $\omega \in \Omega$. Property (iii) ensures that $Q_{\omega}$ is a $p$-group, and so the group $H_{\omega}$ is periodic.

It remains to observe that for every $\omega \in \Omega$, the finitely generated group $G_{\omega}$ can have only finitely many distinct normal subgroups of index $k$, hence among $\left\{G_{\omega} \mid \omega \in \Omega\right\}$ there must be $|\Omega|=2^{\aleph_{0}}$ pairwise non-isomorphic groups.

Proof of Corollary 1. First, we observe that since $G / N$ is a $p$-group, there is $n \in \mathbb{N}$ such that $\delta_{n}^{p}(G) \leq N$ (by the first part of Remark 3). Since $\left|G: \delta_{n}^{p}(G)\right|<\infty$ and since there is an epimorphism $\varphi: N \rightarrow F_{2}$, the subgroup $\varphi\left(\delta_{n}^{p}(G)\right)$ has finite index in $\varphi(N)=F_{2}$. Hence $\delta_{n}^{p}(G) \rightarrow F_{2}$. Thus, without loss of generality, we may suppose that $N=\delta_{n}^{p}(G)$ for some $n \in \mathbb{N}$.

Now we apply Lemma 21 to $G, N=\delta_{n}^{p}(G)$ and $p$, to obtain the groups $H_{\omega}$ as claimed. Since $H_{\omega} / Q_{\omega} \cong G / \delta_{n}^{p}(G)$ is a finite $p$-group, the property (iii) of $Q_{\omega}$ implies that $H_{\omega}$ is a periodic $p$-group. From the proof of Lemma 21 we see that $Q_{\omega}$ is the image of $N$ under the natural homomorphism $G \rightarrow H_{\omega}$. Therefore 
$Q_{\omega}=\delta_{n}^{p}\left(H_{\omega}\right)$ and $\delta_{j}^{p}\left(Q_{\omega}\right)=\delta_{j+n}^{p}\left(H_{\omega}\right)$ for every $j \in \mathbb{N}$. Thus, property (iv) of $Q_{\omega}$ yields property (b) of $H_{\omega}$, and properties (ii) and (v) together yield (c) (for $t=v+n)$.

Proof of Corollary 2. By a theorem of Baumslag and Pride [5], for any prime $p$ there are a normal subgroup $N \triangleleft G$ and $m \in \mathbb{N}$ such that $|G: N|=p^{m}$ and $N \rightarrow F_{2}$. To conclude, it remains to apply Corollary 1 to the pair $(G, N)$.

Acknowledgements. The first and the third authors are grateful to Institut des Hautes Études Scientifiques (IHÉS) for the hospitality during their work on this article. The authors would also like to thank L. Bartholdi and R. I. Grigorchuk for interesting discussions, and the referee for his remarks.

\section{References}

[1] S. I. Adian, The Burnside problem and identities in groups. Ergeb. Math. Grenzgeb. 95, Springer-Verlag, Berlin 1979. Zbl 0417.20001 MR 0537580

[2] J. M. Alonso et al., Notes on word hyperbolic groups (ed. by H. Short). In Group theory from a geometrical viewpoint (Trieste, 1990), World Scientific, Singapore 1991, 3-63. Zbl 0849.20023 MR 1170363

[3] V. S. Atabekyan, Simple and free periodic groups. Vestnik Moskov. Univ. Ser. I Mat. Mekh. 1987 (1987), no. 6, 76-78; English transl. Moscow Univ. Math. Bull. 42 (1987), no. 6, 80-82. Zbl 0661.20027 MR 922890

[4] L. Bartholdi, Grigorchuk's group has locally countably many quotients. Unpublished.

[5] B. Baumslag and S. J. Pride, Groups with two more generators than relators. J. London Math. Soc. (2) 17 (1978), 425-426. Zbl 0387.20030 MR 0491967

[6] N. Bourbaki, Groupes et algèbres de Lie. Chap. IV-VI, Hermann, Paris, 1968. Zbl 0186.33001 MR 0240238

[7] M. R. Bridson and A. Haefliger, Metric spaces of non-positive curvature. Grundlehren Math. Wiss. 319, Springer-Verlag, Berlin 1999. Zbl 0988.53001 MR 1744486

[8] W. Burnside, On an unsettled question in the theory of discontinous groups. Quart. J. Pure and Appl. Math. 33 (1902), 230-238. JFM 33.0149.01

[9] E. Cartan, Leçons sur la géométrie des espaces de Riemann, Gauthier-Villars, Paris 1928. JFM 54.0755.01

[10] G. S. Deryabina, Infinite p-groups with cyclic subgroups. Mat. Sb. (N.S.) 124 (166) (1984), 495-504; English transl. Math. USSR-Sb. 52 (1985), 481-490. Zbl 0556.20032 MR 754473

[11] B. Fine and G. Rosenberger, Algebraic generalizations of discrete groups. Monographs Textbooks Pure Appl. Math. 223, Marcel Dekker, New York 1999. Zbl 0933.20001 MR 1712997

[12] E. Ghys and P. de la Harpe (eds.), Sur les groupes hyperboliques d'après Mikhael Gromov. Progr. Math. 83, Birkhäuser, Boston 1990. Zbl 0731.20025 MR 1086648 
[13] E. S. Golod, On nil-algebras and finitely approximable $p$-groups. Izv. Akad. Nauk SSSR Ser. Mat. 28 (1964), 273-276; English transl. Amer. Math. Soc. Transl. Ser. (2) 48 (1965), 103-106. Zbl 0215.39202 MR 0161878

[14] R. I. Grigorchuk, On Burnside's problem on periodic groups. Funktsional. Anal. i. Prilozhen. 14 (1980), 53-54; English transl. Funct. Anal. Appl. 14 (1980), 41-43. Zbl 0595.20029 MR 0565099

[15] R. I. Grigorchuk, Degrees of growth of finitely generated groups, and the theory of invariant means. Izv. Akad. Nauk SSSR Ser. Mat. 48 (1984), 939-985; English transl. Math. USSR-Izv. 25 (1985), 259-300. Zbl 0583.20023 MR 0764305

[16] R. I. Grigorchuk, On the growth degrees of $p$-groups and torsion-free groups. Mat. Sb. (N.S.) 126 (168) (1985), 194-214; English transl. Math. USSR-Sb. 54 (1986), 185-205. Zbl 0583.20024 MR 0784354

[17] R. I. Grigorchuk, Just infinite branch groups. In New horizons in pro-p groups, Progr. Math. 184, Birkhäuser Boston, Boston 2000, 121-179. Zbl 0982.20024 MR 1765119

[18] M. Gromov, Hyperbolic groups. In Essays in group theory, Math. Sci. Res. Inst. Publ. 8, Springer-Verlag, New York 1987, 75-263. Zbl 0634.20015 MR 0919829

[19] S. V. Ivanov, The free Burnside groups of sufficiently large exponents. Internat. J. Algebra Comput. 4 (1994), 1-308. Zbl 0822.20044 MR 1283947

[20] S. V. Ivanov and A. Yu. Ol'shanskii, Hyperbolic groups and their quotients of bounded exponents. Trans. Amer. Math. Soc. 348 (1996), 2091-2138. Zbl 0876.20023 MR 1327257

[21] I. Kapovich, A non-quasiconvexity embedding theorem for hyperbolic groups. Math. Proc. Cambridge Philos. Soc. 127 (1999), 461-486. Zbl 0942.20026 MR 1713122

[22] I. Kapovich and D. T. Wise, The equivalence of some residual properties of wordhyperbolic groups. J. Algebra 223 (2000), 562-583. Zbl 0951.20029 MR 1735163

[23] M. I. Kargapolov and Yu. I. Merzlyakov, Osnovy teorii grupp. 3rd ed., Nauka, Moscow 1982; English transl. of the 2nd Russian ed. Fundamentals of group theory, Graduate Texts in Math. 62, Springer-Verlag, New York 1979. Zbl 0256.20002 MR 0677282

[24] V. D. Mazurov and E. I. Khukhro (eds.), The Kourovka notebook. Unsolved problems in group theory. Including archive of solved problems. 16th ed., Institute of Mathematics, Novosibirsk 2006. Zbl 1084.20001 MR 2263886

[25] M. Lackenby, Adding high powered relations to large groups. Math. Res. Lett. 14 (2007), 983-993. Zbl 05243868 MR 2357469

[26] R. C. Lyndon and P. E. Schupp, Combinatorial group theory. Ergeb. Math. Grenzgeb. 89, Springer-Verlag, Berlin 1977. Zbl 0368.20023 MR 0577064

[27] I. G. Lysënok, Infinite Burnside groups of even period. Izv. Ross. Akad. Nauk Ser. Mat. 60 (1996), 3-224; English transl. Izv. Math. 60 (1996), 453-654. Zbl 0926.20023 MR 1405529

[28] A. Minasyan, Some properties of subsets of hyperbolic groups. Comm. Algebra 33 (2005), 909-935. Zbl 1080.20036 MR 2128420

[29] V. Nekrashevych, Self-similar groups. Math. Surveys Monogr. 117, Amer. Math. Soc., Providence, RI, 2005. Zbl 1087.20032 MR 2162164 
[30] P. S. Novikov and S. I. Adjan, Infinite periodic groups. I, II, III. Izv. Akad. Nauk SSSR Ser. Mat. 32 (1968), 212-244, 251-524, 709-731; English transl. Math. USSR-Izv. 2 (1968), 209-236, 241-479, 665-685. Zbl 0194.03301 MR 0240178

[31] A. Yu. Ol'shanskii, Groups with cyclic subgroups (Russian). C. R. Acad. Bulgare Sci. 32 (1979), 1165-1166. Zbl 0431.20026 MR 562927

[32] A. Yu. Ol'shanskii, Geometriya opredelyayushchikh sootnoshenij v gruppakh. Nauka, Moscow 1989; English transl. Geometry of defining relations in groups. Math. Appl. (Soviet Ser.) 70, Kluwer Academic Publishers, Dordrecht 1991. Zbl 0676.20014 MR 1024791

[33] A. Yu. Ol'shanskiǔ, Periodic factor groups of hyperbolic groups. Mat. Sb. 182 (1991), 543-567; Math. USSR-Sb. 72 (1992), 519-541. Zbl 0820.20044 MR 1119008

[34] A. Yu. $\mathrm{Ol}^{\prime}$ shanskii, On residualing homomorphisms and $G$-subgroups of hyperbolic groups. Internat. J. Algebra Comput. 3 (1993), 365-409. Zbl 0830.20053 MR 1250244

[35] A. Yu. Ol'shanskii, The growth of finite subgroups in p-groups. In Groups St. Andrews 1997 in Bath, II. London Math. Soc. Lecture Note Ser. 261, Cambridge University Press, Cambridge 1999, 579-595. Zbl 0938.20031 MR 1676655

[36] A. Yu. Ol'shanskii, On the Bass-Lubotzky question about quotients of hyperbolic groups. J. Algebra 226 (2000), 807-817. Zbl 0957.20026 MR 1752761

[37] A. Yu. Olshanskii and D. V. Osin, Large groups and their periodic quotients. Proc. Amer. Math. Soc. 136 (2008), 753-759. Zbl 1151.20032 MR 2361846

[38] D. Sonkin, On simple groups of large exponents. Algebra Discrete Math. 2004 (2004), no. 4, 79-105. Zbl 1092.20037 MR 2148719

[39] E. I. Zel'manov, Solution of the restricted Burnside problem for groups of odd exponent. Izv. Akad. Nauk SSSR Ser. Mat. 54 (1990), 42-59; English transl. Math. USSR-Izv. 36 (1991), 41-60. Zbl 0704.20030 MR 1044047

[40] E. I. Zel'manov, Solution of the restricted Burnside problem for 2-groups. Mat. Sb. 182 (1991), 568-592; English transl. Math. USSR-Sb. 72 (1992), 543-565. Zbl 0752.20017 MR 1119009

Received April 22, 2008; revised October 26, 2008

A. Minasyan, School of Mathematics, University of Southampton, Highfield, Southampton, SO17 1BJ, UK

E-mail: aminasyan@gmail.com

A. Yu. Olshanskii, Department of Mathematics, 1326 Stevenson Center, Vanderbilt

University Nashville, TN 37240, U.S.A. and

Department of Mechanics and Mathematics, Moscow State University, Moscow, 119899,

Russia

E-mail: alexander.olshanskiy@vanderbilt.edu

D. Sonkin, Department of Mathematics, P.O. Box 400137 University of Virginia,

Charlottesville, VA 22904, U.S.A.

E-mail: ds5nd@virginia.edu 